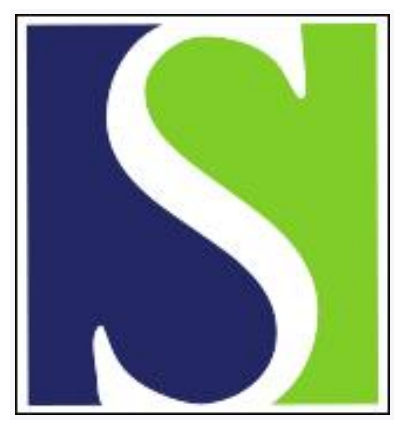

Scand J Work Environ Health 2015;41(5):425-440

https://doi.org/10.5271/sjweh.3509

Published online: 23 Jun 2015, Issue date: 01 Sep 2015

A systematic review of the sleep, sleepiness, and performance implications of limited wake shift work schedules

by Short MA, Agostini A, Lushington K, Dorrian J

This paper reviews the implications of limited wake shift work schedules, such as the $6 \mathrm{hr}$-on/6hr-off and $4 \mathrm{hr}$-on/8hr-off watchkeeping schedules employed by mariners and the $8 \mathrm{hr}$-on/8hr-off schedules of long-haul train drivers. These schedules are reviewed within a chronobiological framework and recommendations are made to optimize sleep and performance.

Affiliation: Centre for Sleep Research, University of South Australia, GPO Box 2100, Adelaide SA 5001, Australia. michelle.short@unisa.edu.au

Refers to the following text of the Journal: 2006;32(3):232-240

Key terms: continuous operation; limited wake shift work; limited wake shift work schedule; performance; performance implication; review; shift work; shift work schedule; sleep; sleepiness; split shift; split sleep; sustained operation; systematic review

This article in PubMed: www.ncbi.nlm.nih.gov/pubmed/26103467 


\title{
A systematic review of the sleep, sleepiness, and performance implications of limited wake shift work schedules
}

\author{
by Michelle A Short, PhD, ${ }^{1,2,3}$ Alexandra Agostini, BPsycSci(Hons), ${ }^{1}$ Kurt Lushington, PhD, ${ }^{1}$ Jillian \\ Dorrian, $P h D^{1}$
}

\begin{abstract}
Short MA, Agostini AA, Lushington K, Dorrian J. A systematic review of the sleep, sleepiness and performance implications of limited wake shift work schedules. Scand J Work Environ Health. 2015;41(5):425-440. doi:10.5271/sjweh.3509
\end{abstract}

Objectives The aim of this review was to identify which limited wake shift work schedules (LWSW) best promote sleep, alertness, and performance. LWSW are fixed work/rest cycles where the time-at-work does is $\leq 8$ hours and there is $>1$ rest period per day, on average, for $\geq 2$ consecutive days. These schedules are commonly used in safety-critical industries such as transport and maritime industries.

Methods Literature was sourced using PubMed, Embase, PsycInfo, Scopus, and Google Scholar databases. We identified 20 independent studies (plus a further 2 overlapping studies), including 5 laboratory and 17 field-based studies focused on maritime watch keepers, ship bridge officers, and long-haul train drivers. The measurement of outcome measures was varied, incorporating subjective and objective measures of sleep: sleep diaries $(\mathrm{N}=5)$, actigraphy $(\mathrm{N}=4)$, and polysomnography, $(\mathrm{N}=3)$; sleepiness: Karolinska Sleepiness Scale $(\mathrm{N}=5)$, visual analog scale (VAS) alertness ( $\mathrm{N}=2$ ) and author-derived measures $(\mathrm{N}=2)$; and performance: Psychomotor Vigilance Test (PVT) $(\mathrm{N}=5)$, Reaction Time or Vigilance tasks $(\mathrm{N}=4)$, Vector and Letter Cancellation Test $(\mathrm{N}=1)$, and subjective performance $(\mathrm{N}=2)$.

Results Of the three primary rosters examined ( 6 hours-on/ 6 hours-off, 8 hours-on/ 8 hours-off and 4 hourson $/ 8$ hours-off), the 4 hours-on/ 8 hours-off roster was associated with better sleep and lower levels of sleepiness. Individuals working 4 hours-on/ 8 hours-off rosters averaged 1 hour more sleep per night than those working 6 hours-on/6 hours-off and 1.3 hours more sleep than those working 8 hours-on/ 8 hours-off $(\mathrm{P}<0.01)$. More broadly, findings indicate that LWSW schedules were associated with better sleep and lower sleepines in the case of (i) shorter time-at-work, (ii) more frequent rest breaks, (iii) shifts that start and end at the same clock time every 24 hours, and (iv) work shifts commencing in the daytime (as opposed to night). The findings for performance remain incomplete due to the small number of studies containing a performance measure and the heterogeneity of performance measures within those that did.

Conclusion The literature supports the utility of LWSW in industries where individuals sleep at or near the workplace as they facilitate at least some sleep during the biological night and minimize deficits associated with time-on-shift with shorter shifts. Overall, the 4 hour-on/8 hour-off roster best promoted sleep and minimized sleepiness compared to other LWSW schedules. Nevertheless, and considering the safety-critical nature of industries which employ LWSW, the limited literature needs to be greatly expanded with specific focus on the consequences for performance and comparison to mainstream rosters.

Key terms continuous operation; performance; split shift; split sleep; sustained operation.

Many industries require continuous, around-the-clock operations, for example, defense, emergency services, health, manufacturing, mining and transport, as well as more rarefied occupations such as space operations (1-6). These industries face a challenge in how to prevent the decrements in safety, alertness, and per- formance that arise from spending a prolonged time at work and working nights. One strategy has been to redistribute time-at-work into smaller aliquots that are spaced equally across the 24-hour period. Commonly employed short cycling work/rest routines or limited wake shift work (LWSW) schedules include 6 hours-

1 Centre for Sleep Research, University of South Australia, Adelaide, Australia.

2 Bushfire Cooperative Research Centre, East Melbourne, Melbourne, Australia.

3 School of Psychology, Flinders University, Adelaide, South Australia.

Correspondence to: Michelle A Short, Centre for Sleep Research, University of South Australia, GPO Box 2100, Adelaide SA 5001, Australia. [E-mail: michelle.short@unisa.edu.au] 
on/ 6 hours-off, 4 hours-on/ 8 hours-off and 8 hours-on/ 8 hours-off schedules. These types of shift schedules minimize the number of consecutive hours at work, promote rest opportunities between work bouts, and allow for at least some opportunity for sleep during the night for all workers (7-11). Given the safety-critical nature of many of the industries that employ LWSW and the emerging research activity in this field, a review of the current literature is timely. In particular, there is a need to identify which LWSW schedules best promote sleep, minimize sleepiness, and facilitate performance.

There is a general consensus that the impact of shiftwork on sleep, sleepiness, and performance is mediated by three factors: (i) time-of-day or circadian effects; (ii) the length of time-at-work (and conversely the opportunity for sleep); and (iii) the consistency of start and finish times $(8,12,13)$. Taking each of these factors in turn, the impact of the circadian system is such that the maximum drive for sleep, the highest levels of sleepiness, and lowest performance tend to occur during the early hours of the morning $(12,14)$ also known as the circadian nadir (15). By contrast, the likelihood of sleep and, likewise, sleepiness are at their lowest in the early evening period also known as the "forbidden sleep zone" or the "wake maintenance zone" (WMZ) (16). Sleep, sleepiness, and performance are also strongly influenced by the length of time spent awake and, in turn, the opportunity for sleep, which are primarily dictated by shift length and the timing of shift cycles (8). Generally, shift schedules that possess a high work-torest ratio (ie, where workers spend a longer time at work and have shorter times between shift) are associated with poorer circadian alignment and shorter sleep (17). Likewise shift schedules where start and finish times are inconsistent from one 24-hour cycle to the next and, as a consequence, the timing work/rest bouts $(18,19)$.

While the factors identified above are relatively well explored in more commonly worked shiftwork schedules (eg, 8 hours-on/16 hours-off), they have received less attention in LWSW. Nevertheless, it is expected that the same trends will be evident. Specifically, it is hypothesized that LWSW schedules will be associated with shorter sleep, greater sleepiness and poorer performance where (i) more of the work time falls across the circadian nadir, (ii) more of the sleep opportunity falls during the WMZ, (iii) the work-to-rest ratio is high, and (iv) the consistency in the timing of work/rest bouts from one 24-hour cycle to the next is low $(20,21)$. The aim of this review is to test these hypotheses and determine which LWSW schedules best promote sleep, alertness, and performance. Given the differences across studies in schedules and methodology, where possible, these hypotheses were tested using meta-analyses (regression modelling). Where available study measures render analysis unfeasible, narrative review of studies were conducted.

\section{Method}

\section{LWSW definition}

A significant challenge in accessing LWSW literature is the lack of a universally accepted definition, or indeed terminology, to describe roster schedules that employ short sleep/wake rotations. Industry-specific conventions include "watchstanding" (marine), "relay" (rail), "twoup" (rail and road), and "platoon" (emergency services). For the purpose of this review, LWSW is defined as "fixed shift work schedules that occur for two or more consecutive days where individuals work for periods not exceeding 8 hours on rosters that allow for an average of more than one sleep period per day". This definition includes roster schedules such as 6 hours-on/6 hours-off, 8 hours-on/ 8 hours-off and 4 hours-on/ 8 hours-off, but excludes those with a single sleep period per 24 hours, such as 12 hours-on/12 hours-off, 16 hours-on/ 8 hoursoff and 8 hours-on/16 hours-off.

\section{Search and inclusion criteria}

Based on the above definition, the relevant literature for this review was sourced using PubMed, Embase, PsycInfo and Scopus databases using the keywords "split sleep", "sustained operations", "extended operations", and "continuous operations," and by inspection of reference lists. Google Scholar was searched with keywords "relay sleep work", "relay sleep work rail", "two-up sleep", "watchstanding sleep", "police platoon shiftwork sleep", "4 hours-on 4 hours-off sleep", "4 hours-on 8 hours-off sleep", "6 hours-on 6 hours-off sleep", " 8 hours-on 8 hours-off sleep". The authors also emailed members of the Working Time Society to request any relevant published studies. All relevant papers were available without contact from the authors. Only primary, peer-reviewed articles published in English were included, while studies examining pharmacological interventions were excluded. From this search, we identified 22 papers which examined LWSW (17-19, 22-40), two pairs of which overlap $(25,29,36,37)$, leaving a total of 20 independent studies. The identified studies are summarized in table 1. A notable feature of the current literature is the large variation in study design, participant groups/industry, roster schedules and outcome variables - a limitation also noted in systematic reviews of other fields of shift work (41-43).

\section{Schedules and participants}

The studies examined in this review employed a range of schedules, which can be broadly divided into: (i) work/rest schedules, such as 4 hours-on/ 8 hours-off, 6 hours-on/ 6 hours-off and 8 hours-on/ 8 hours-off sched- 
Table 1. Study characteristics and key results of limited wake shift work (LWSW) studies. Far right columns show evaluation of span of evidence in the studies for the purposes of this review. [SLP=sleep measures ( $0=$ not measured; $1=$ diary; $2=a c t i g r a p h y ; 3=P S G$ ); PERF=performance measures ( $0=$ not measured; 1 =subjective measurements; $2=0$ jective measurements); SLPY=sleepiness/alertness ( $0=$ not measured; 1 =subjective measurements; 2=objective measurements); TOT=total of SLP, PERF, and SLPY (possible total=7); $\mathrm{CBT}=$ core body temperature; DSST=digit symbol substitution test; ESS=Epworth sleepiness scale; KSS=Karolinska sleepiness scale; $M E Q=$ morningness-eveningness questionnaire; NR=not reported; PANAS=positive affect negative affect schedule; POMS=profile of mood states; PSG=polysomnography; PVT=psychomotor vigilance test; SE=sleep efficiency; SOL=sleep onset latency; $S S Q=s u b j e c t i v e ~ s l e e p$ quality; SSS=Stanford sleepiness scale; SWS=slow wave sleep; REM=rapid eye movement sleep; TIB=time in bed; TST=total sleep time; VAS-M=visual analog scale of mood].

\begin{tabular}{|c|c|c|c|c|c|c|c|c|c|}
\hline Study & $\begin{array}{l}\mathrm{N}, \text { mean }(\mathrm{SD}) \\
\text { age \& range }\end{array}$ & $\begin{array}{l}\text { Operation and } \\
\text { schedule }\end{array}$ & Measures & Design & Results & SLP & PER & SLP & TOT \\
\hline $\begin{array}{l}\text { Arendt et al } \\
2006(26)\end{array}$ & $\begin{array}{l}14 \text { watch } \\
\text { keepers and } \\
12 \text { day } \\
\text { workers } \\
25 \text { male, } \\
1 \text { female } \\
37.8(10.8) \\
\text { years }\end{array}$ & $\begin{array}{l}\text { 3-4 week sea } \\
\text { journeys } \\
\text { Watch keepers } \\
4 \mathrm{~h}-\text { on/8h-off } \\
8 \text { watch keepers } \\
\text { rotated shifts by } \\
\text { delay weekly }\end{array}$ & $\begin{array}{l}\text { Sleep diary } \\
\text { Actigraphy } \\
\text { Urinary } \\
\text { melatonin }\end{array}$ & $\begin{array}{l}\text { (i) Comparison of } \\
\text { day workers and } 3 \\
\text { watch-keeping shifts } \\
\text { and (ii) comparison of } \\
\text { fixed watch schedule } \\
\text { with rotating watch } \\
\text { schedule }\end{array}$ & $\begin{array}{l}\text { Objective SE and SOL was worse in rota- } \\
\text { tors compared to fixed and day workers, } \\
\text { although TST was longer } \\
\text { Second sleep was less efficient than } \\
\text { main (night) sleep for all watch keepers } \\
\text { Sleep similar across all watch schedules. } \\
\text { Efficiency < } 80 \% \text { for all workers } \\
\text { Circadian timing was later in } 0000 \mathrm{~h}- \\
\text { 0400h watch keepers }\end{array}$ & 2 & 0 & 0 & 2 \\
\hline $\begin{array}{l}\text { Condon et } \\
\text { al } 1984 \\
\text { (35) }\end{array}$ & $\begin{array}{l}9 \text { watch } \\
\text { keepers and } \\
3 \text { day workers } \\
\text { Age } 25-44 \\
\text { years }\end{array}$ & $\begin{array}{l}\text { 4h-on/4h-off } \\
24 \mathrm{~h} \text { watch } \\
\text { schedule }\end{array}$ & $\begin{array}{l}\text { Oral temp- } \\
\text { erature after } \\
\text { several weeks } \\
\text { of schedule }\end{array}$ & $\begin{array}{l}\text { Comparison of tem- } \\
\text { perature curve be- } \\
\text { tween shift schedules }\end{array}$ & $\begin{array}{l}\text { Oral temperature curve showed adapta- } \\
\text { tion to shift. Earliest acrophase seen } \\
\text { in the } 04: 00 \mathrm{~h}-08: 00 \mathrm{~h} / 16: 00 \mathrm{~h}-20: 00 \mathrm{~h} \\
\text { watchkeeping shift workers and day } \\
\text { workers. Acrophase was relatively late } \\
\text { for } 00: 00 \mathrm{~h}-04: 00 \mathrm{~h} / 12: 00 \mathrm{~h}-16: 00 \mathrm{~h} \text { shift. }\end{array}$ & 0 & 0 & 0 & 0 \\
\hline $\begin{array}{l}\text { Condon et } \\
\text { al } 1988 \\
\text { (27) }\end{array}$ & $\begin{array}{l}19 \text { watch } \\
\text { keepers and } \\
20 \text { day } \\
\text { workers } \\
\text { Age NR }\end{array}$ & $\begin{array}{l}\text { 4h-on/8h-off } \\
24 \mathrm{~h} \text { watch } \\
\text { schedule }\end{array}$ & $\begin{array}{l}\text { Vector Test } \\
\text { Letter } \\
\text { Cancellation } \\
\text { Test } \\
\text { VAS alertness }\end{array}$ & $\begin{array}{l}\text { Compare the type } \\
\text { and level of circadian } \\
\text { variation in alertness } \\
\text { and performance } \\
\text { across different shift } \\
\text { schedules (04-08/16- } \\
20,12-16 / 00-04 \text {, } \\
08-12 / 20-24)\end{array}$ & $\begin{array}{l}\text { Alertness increased from beginning end } \\
\text { first shift per day and decreased for sec- } \\
\text { ond shift. } \\
\text { Vector accuracy increased from begin- } \\
\text { ning to end of both shifts. } \\
\text { Vector speed showed the same pattern } \\
\text { for those working } 08-12 / 20-24 \text { but de- } \\
\text { creased during the second shift for those } \\
\text { on the other schedules. }\end{array}$ & 0 & 2 & 1 & 4 \\
\hline $\begin{array}{l}\text { Colquhoun } \\
\text { et al, } 1968 \\
\text { (38) }\end{array}$ & $\begin{array}{l}12 \text { male } \\
\text { participants } \\
\text { Age NR }\end{array}$ & $\begin{array}{l}\text { 4-crew rotating } \\
\text { schedule: } 4 \mathrm{~h}- \\
\text { on/4h-off/ } \\
4 \mathrm{~h}-0 \mathrm{n} / 8 \mathrm{~h}-\mathrm{off} / \\
4 \mathrm{~h}-\mathrm{on} / 14 \mathrm{~h}-\mathrm{off} / \\
4 \mathrm{~h}-\mathrm{on} / 4 \mathrm{~h}-\mathrm{off}\end{array}$ & $\begin{array}{l}\text { Oral } \\
\text { temperature } \\
\text { Auditory vigi- } \\
\text { lance test }\end{array}$ & $\begin{array}{l}\text { Description of the } \\
\text { circadian rhythms of } \\
\text { temperature and per- } \\
\text { formance across a ro- } \\
\text { tating 4-crew schedule } \\
\text { for } 11-12 \text { days }\end{array}$ & $\begin{array}{l}\text { Temperature rhythm did not show any } \\
\text { adaptation across } 4 \text { days of this rotating } \\
\text { schedule. Auditory vigilance perfor- } \\
\text { mance closely followed the circadian } \\
\text { temperature rhythm. } \\
\text { Interpretation of findings limited by re- } \\
\text { versal of the Day } 2 \text { and } 3 \text { schedules for } \\
\text { half of the sample }\end{array}$ & 0 & 2 & 0 & 2 \\
\hline $\begin{array}{l}\text { Colquhoun } \\
\text { et al, } 1987 \\
\text { (18) }\end{array}$ & $\begin{array}{l}6 \text { watch } \\
\text { keepers on } \\
\text { a naval ship } \\
\text { Age NR }\end{array}$ & $\begin{array}{l}\text { 4-day schedule: } \\
\text { 4h-on/12h-off/2h- } \\
\text { on/10h-off/2h- } \\
\text { on/12h-off/4h- } \\
\text { on/8h-off/4h- } \\
\text { on } / 12 \mathrm{~h}-\mathrm{off} / 4 \mathrm{~h}- \\
\text { on } / 8 \mathrm{~h}-\mathrm{off}\end{array}$ & $\begin{array}{l}\text { Oral } \\
\text { temperature } \\
\text { Auditory } \\
\text { vigilance test } \\
\text { VAS alertness } \\
\text { Activity log }\end{array}$ & $\begin{array}{l}\text { Description of the } \\
\text { sleep, work, perfor- } \\
\text { mance and tempera- } \\
\text { ture rhythms of watch- } \\
\text { keepers over a 4-day } \\
\text { shift schedule after } \\
\text { several months at sea }\end{array}$ & $\begin{array}{l}\text { TST averaged } 7.7 \text { per day } \\
\text { No obvious changes in temperature } \\
\text { rhythm } \\
\text { VAS alertness lowest during night } \\
\text { watches } \\
\text { Daytime alertness lowest following night } \\
\text { watches }\end{array}$ & 1 & 2 & 1 & 4 \\
\hline $\begin{array}{l}\text { Darwent et } \\
\text { al } 2008 \text { (22) }\end{array}$ & $\begin{array}{l}10 \text { male train } \\
\text { drivers } \\
43.3(7.4) \\
\text { years; } \\
32-53 \text { years }\end{array}$ & $\begin{array}{l}\text { 106h operation } \\
8 \mathrm{~h}-\mathrm{on} / 8 \mathrm{~h}-\mathrm{off}\end{array}$ & $\begin{array}{l}\text { Sleep diary } \\
\text { SSQ } \\
\text { Actigraphy } \\
\text { PVT }\end{array}$ & $\begin{array}{l}\text { (i) Comparison of } \\
\text { sleep environments } \\
\text { (home vs. crew van } \\
\text { vs. hotel layover) and } \\
\text { (ii) comparison of } \\
\text { shift rotation (team } \\
\text { one commenced duty } \\
\text { at } 0800-1600 \mathrm{~h} \text { [early] } \\
\text { and team two at } 1600- \\
2400 \mathrm{~h} \text { [late]) } \\
8-12 \mathrm{~h} \text { layover halfway } \\
\text { through the rotation, } \\
\text { but due to the variabil- } \\
\text { ity in layover duration, } \\
\text { the start time of post- } \\
\text { layover shifts varied } \\
\text { between drivers }\end{array}$ & $\begin{array}{l}\text { Cumulative sleep loss not different be- } \\
\text { tween rotations but did increase across } \\
\text { subsequent trip days } \\
\text { TIB, TST \& SE lower on early rotation } \\
\text { TIB, TST \& SE lower in crew van com- } \\
\text { pared to home and layover sites } \\
\text { SSQ similar in crew van, at home and } \\
\text { layover sites } \\
\text { PVT poorer on late rotation } \\
\text { PVT constant from shift start to end }\end{array}$ & 2 & 2 & 0 & 4 \\
\hline
\end{tabular}


Table 1. continued

\begin{tabular}{|c|c|c|c|c|c|c|c|c|c|}
\hline Author/Date & $\begin{array}{l}\text { No, mean }(S D) \\
\text { age \& range }\end{array}$ & $\begin{array}{l}\text { Operation and } \\
\text { schedule }\end{array}$ & Measures & Design & Results & SLP & PEF & $\mathrm{SLF}$ & TOT \\
\hline \multirow{6}{*}{$\begin{array}{l}\text { Eriksen et al } \\
2006(23)\end{array}$} & \multirow{3}{*}{$\begin{array}{l}12 \text { male } \\
\text { merchant ma- } \\
\text { rine and navy } \\
\text { navigators }\end{array}$} & \multirow{6}{*}{$\begin{array}{l}\text { 66h operation } \\
6 \text { h-on/6h-off }\end{array}$} & \multirow{6}{*}{$\begin{array}{l}\text { KSS } \\
\text { Sleep diary }\end{array}$} & \multirow{3}{*}{$\begin{array}{l}\text { Comparison of shift } \\
\text { rotation (team one } \\
0600-1200 \mathrm{~h} \text { and team } \\
\text { two } 1200-1800 \mathrm{~h} \text { ) }\end{array}$} & \multirow{2}{*}{$\begin{array}{l}\text { Subjective sleepiness and, likewise, } \\
\text { sleep duration was similar for each } \\
\text { watch rotation }\end{array}$} & \multirow[t]{6}{*}{1} & \multirow[t]{6}{*}{0} & \multirow[t]{6}{*}{1} & \multirow[t]{6}{*}{2} \\
\hline & & & & & & & & & \\
\hline & & & & & \multirow{2}{*}{$\begin{array}{l}\text { Subjective sleepiness was higher on the } \\
\text { night }(2400-0600 \mathrm{~h}) \text { than both the day } \\
(1200-1800 \mathrm{~h}) \text { and evening }(1800-2400 \mathrm{~h}) \\
\text { watches }\end{array}$} & & & & \\
\hline & \multirow[t]{3}{*}{$\begin{array}{l}39.0(N R) \\
\text { years; } \\
26-51 \text { years }\end{array}$} & & & \multirow{3}{*}{$\begin{array}{l}2 \times 3 \mathrm{~h} \text { dog watches } \\
\text { scheduled half way } \\
\text { through the rotation at } \\
1200-1800 \mathrm{~h} \text { resulting } \\
\text { in reversal of on- and } \\
\text { off-duty patterns }\end{array}$} & & & & & \\
\hline & & & & & $\begin{array}{l}\text { Subjective sleepiness increased } \\
\text { from beginning to end of each watch } \\
(1200-1800 \mathrm{~h}, 1800-2400 \mathrm{~h} \& 2400- \\
0600 \mathrm{~h}) \text { except for the morning watch } \\
(0600-1200 \mathrm{~h})\end{array}$ & & & & \\
\hline & & & & & $\begin{array}{l}\text { Sleep duration was longer on the morn- } \\
\text { ing }(0600-1200 \mathrm{~h}) \text { than the day (1200- } \\
1800 \mathrm{~h}) \text { off-duty watches and longer on } \\
\text { the night than both the day and evening } \\
\text { off-duty watches }\end{array}$ & & & & \\
\hline \multirow[t]{3}{*}{$\begin{array}{l}\text { Hansen et al } \\
2011 \text { (34) }\end{array}$} & \multirow{3}{*}{$\begin{array}{l}577 \text { work- } \\
\text { ers from a } \\
\text { Norwegian } \\
\text { based shipping } \\
\text { company ( } 362 \\
\text { working 6h- } \\
\text { on/6h-off, } 182 \\
\text { working } 12 \mathrm{~h}- \\
\text { on/12h-off). } \\
\text { Age NR }\end{array}$} & \multirow[t]{3}{*}{ 6h-on/6h-off } & \multirow[t]{3}{*}{$\begin{array}{l}\text { Questionnaire- } \\
\text { based survey } \\
\text { focusing on } \\
\text { sleep and } \\
\text { shiftwork }\end{array}$} & \multirow[t]{3}{*}{$\begin{array}{l}\text { Comparison of } 6 \mathrm{~h}- \\
\text { on/6h-off and } 12 \mathrm{~h}- \\
\text { on } 12 \mathrm{~h}-\mathrm{off} \text { roster }\end{array}$} & $\begin{array}{l}\text { Workers on } 6 \mathrm{~h} \text {-on/6h-off schedule re- } \\
\text { ported more sleep disturbances than did } \\
\text { workers on } 12 \mathrm{~h} \text {-on } / 12 \mathrm{~h} \text {-off schedule. } \\
\text { Causes were similar between groups, } \\
\text { however, noise affected the } 6 \text {-h-on/6h- } \\
\text { off group more and other factors more } \\
\text { greatly affected the } 12 \mathrm{~h} \text {-on/12h-off } \\
\text { group. }\end{array}$ & \multirow[t]{3}{*}{1} & \multirow[t]{3}{*}{1} & \multirow[t]{3}{*}{0} & \multirow[t]{3}{*}{2} \\
\hline & & & & & $\begin{array}{l}\text { No group differences in work capability } \\
\text { or safety. }\end{array}$ & & & & \\
\hline & & & & & $\begin{array}{l}\text { Most workers in both groups do not } \\
\text { have the same sleep disturbances at } \\
\text { home, suggesting disturbances were } \\
\text { caused by the work itself. }\end{array}$ & & & & \\
\hline \multirow{8}{*}{$\begin{array}{l}\text { Härma et al } \\
2008 \text { (17) }\end{array}$} & \multirow{4}{*}{$\begin{array}{l}185 \text { bridge } \\
\text { officers } \\
6 \mathrm{~h}-\text { on } / 6 \mathrm{~h}-\text { off } \\
=45.1(9.8) \\
\text { years }\end{array}$} & \multirow{3}{*}{$\begin{array}{l}168 \mathrm{~h} \\
6 \mathrm{~h}-\mathrm{on} / 6-\mathrm{off} \\
(\mathrm{N}=45)\end{array}$} & \multirow{5}{*}{$\begin{array}{l}\text { KSS } \\
\text { EPS } \\
\text { Skogby } \\
\text { Excessive } \\
\text { Daytime } \\
\text { Sleepiness } \\
\text { Index }\end{array}$} & \multirow{3}{*}{$\begin{array}{l}\text { Comparison of (i) } \\
\text { shift rotation and (ii) } \\
\text { systems }\end{array}$} & \multirow{2}{*}{$\begin{array}{l}\text { 6h-on/6-off : TST was similar for both } \\
\text { watch rotations }\end{array}$} & \multirow[t]{8}{*}{1} & \multirow[t]{8}{*}{0} & \multirow[t]{8}{*}{1} & \multirow[t]{8}{*}{2} \\
\hline & & & & & & & & & \\
\hline & & & & & 4h-on/8h-off: TST was higher for team & & & & \\
\hline & & $\begin{array}{l}\text { 4h-on/8h-off } \\
(\mathrm{N}=68)\end{array}$ & & $\begin{array}{l}6 \mathrm{~h}-\mathrm{on} / \mathrm{6}-\mathrm{off} \text { team one } \\
\text { commenced duty at }\end{array}$ & $\begin{array}{l}\text { one followed by team three then team } \\
\text { two watch rotations }\end{array}$ & & & & \\
\hline & $\begin{array}{l}4 \mathrm{~h} \text {-on } / 8 \mathrm{~h}-\mathrm{off} \\
=36.9(9.8) \\
\text { years }\end{array}$ & $\begin{array}{l}\text { Other combi- } \\
\text { nations (e.g. }\end{array}$ & & $\begin{array}{l}0600-1200 \mathrm{~h} \text { and team } \\
\text { two at } 1200-1800 \mathrm{~h} ; \\
\text { and }\end{array}$ & $\begin{array}{l}\text { TST was shorter, frequency of nodding } \\
\text { while on duty and excessive daytime } \\
\text { sleepiness was higher on } 6 \mathrm{~h}-0 \mathrm{n} / 6 \text { - }\end{array}$ & & & & \\
\hline & $\begin{array}{l}\text { Other combi- } \\
\text { nations }=42.9\end{array}$ & $\begin{array}{l}4 \mathrm{~h}-0 \mathrm{n} / 4 \mathrm{~h}-\mathrm{off}, \mathrm{etc}) \\
(\mathrm{N}=52)\end{array}$ & seep alary & & $\begin{array}{l}\text { off compared to } 4 \mathrm{~h}-\mathrm{on} / 8 \mathrm{~h}-\mathrm{off} \text { watch } \\
\text { systems }\end{array}$ & & & & \\
\hline & (12.3) years & & & $\begin{array}{l}\text { duty at } 0000-0400 \mathrm{~h}, \\
\text { team two at } 0400- \\
0800 \mathrm{~h} \text { and team three } \\
0800-1200 \mathrm{~h}\end{array}$ & $\begin{array}{l}\text { Time-of-day, time spent awake, and } \\
\text { sleep length but not watch system did } \\
\text { not significantly predicted sleepiness } \\
\text { during watch duty }\end{array}$ & & & & \\
\hline & & & & $\begin{array}{l}\text { The last seven days at } \\
\text { sea were used in the } \\
\text { analyses }\end{array}$ & $\begin{array}{l}\text { History of snoring was more strongly } \\
\text { predictive of excessive daytime sleepi- } \\
\text { ness than watch system }\end{array}$ & & & & \\
\hline Hartley 1974 & 36 male & $96 \mathrm{~h}$ & Visual vigi- & Comparison of an- & Visual vigilance was higher after $8 \mathrm{~h}$ & 0 & 2 & 0 & 2 \\
\hline & & 8h sleep, 4h sleep & lance test & $\begin{array}{l}\text { chored vs. distributed } \\
\text { sleep. }\end{array}$ & $\begin{array}{l}\text { nocturnal sleep followed by distributed } \\
3 \times 80 \text { min naps then } 4 \mathrm{~h} \text { nocturnal sleep }\end{array}$ & & & & \\
\hline & & & & $\begin{array}{l}\text { Team one had } 8 \mathrm{~h} \text { noc- } \\
\text { turnal sleep }(2300 \mathrm{~h}- \\
0700 \mathrm{~h}) \text {, team two had } \\
4 \mathrm{~h} \text { nocturnal sleep } \\
\text { (0100h-0500h) and } \\
\text { team } 3 \text { had } 3 \times 80 \mathrm{~min} \\
\text { naps }(2310 \mathrm{~h}-0030 \mathrm{~h} ; \\
0530 \mathrm{~h}-0650 \mathrm{~h} \& \\
1225 \mathrm{~h}-1345 \mathrm{~h})\end{array}$ & & & & & \\
\hline $\begin{array}{l}\text { Howarth et al } \\
1999(28)\end{array}$ & $\begin{array}{l}138 \text { commer- } \\
\text { cial maritime } \\
\text { crewmembers. }\end{array}$ & $\begin{array}{l}\text { Commercial crew- } \\
\text { members worked } \\
4 \text { h-on/8h-off or }\end{array}$ & $\begin{array}{l}\text { Sleep diary } \\
\text { Items derived } \\
\text { from Standard }\end{array}$ & $\begin{array}{l}\text { Compare severity of } \\
\text { sleep problems on- } \\
\text { duty and off-duty }\end{array}$ & $\begin{array}{l}\text { When off-duty, commercial crewmem- } \\
\text { bers reported significantly less sleep } \\
\text { problems than when on-duty. }\end{array}$ & 1 & 0 & 0 & 1 \\
\hline & $\begin{array}{l}118 \text { male, } 8 \\
\text { female. Mean } \\
\text { age } 41.8 \text { years. }\end{array}$ & & $\begin{array}{l}\text { Shiftwork } \\
\text { Index and } \\
\text { Work-Sleep } \\
\text { Survey }\end{array}$ & & $\begin{array}{l}\text { Sleep length greater while off-duty } \\
\text { ( } 478.41 \text { minutes) than while on-duty } \\
\text { (441.01 minutes). }\end{array}$ & & & & \\
\hline
\end{tabular}


Table 1. continued

\begin{tabular}{|c|c|c|c|c|c|c|c|c|c|}
\hline Author/Date & & & Measures & Design & Results & SLP & PEF & SL & TOT \\
\hline $\begin{array}{l}\text { Jackson et } \\
\text { al, } 2014 \text { (39) }\end{array}$ & $\begin{array}{l}52 \text { healthy } \\
\text { male } \\
\text { participants } \\
26.51(4.07) \\
\text { years; } 22-40 .\end{array}$ & $\begin{array}{l}19 \text { participants } \\
\text { consolidated } 10 \mathrm{~h} \\
\text { TIB night time } \\
\text { sleep, } 17 \text { partici- } \\
\text { pants split } \\
\text { sleep (TIB 0300- } \\
0800 \text { and } 1500- \\
\text { 2000), } 17 \text { partici- } \\
\text { pants consolidated } \\
\text { 10h TIB daytime } \\
\text { sleep }\end{array}$ & $\begin{array}{l}\text { PVT } \\
\text { DSST } \\
\text { KSS } \\
\text { PSG } \\
\text { VAS-Mood } \\
\text { PANAS }\end{array}$ & $\begin{array}{l}\text { Compare three sleep } \\
\text { conditions based on } \\
\text { TST, performance and } \\
\text { mood. }\end{array}$ & $\begin{array}{l}\text { Participants in the night time sleep con- } \\
\text { dition obtained the most sleep, followed } \\
\text { by those in the split sleep condition } \\
\text { (greater TST in the morning naps com- } \\
\text { pared to afternoon naps), followed by } \\
\text { those in the daytime sleep condition. } \\
\text { No significant difference in DSST, PVT } \\
\text { lapses or fastest } 10 \% \text { of responses } \\
\text { between conditions, however, partici- } \\
\text { pants in daytime sleep condition were } \\
\text { significantly sleepier than those in split } \\
\text { sleep or night time sleep conditions. } \\
\text { Participants in split sleep condition had } \\
\text { subjectively lower mood than those in } \\
\text { the daytime or night time sleep condi- } \\
\text { tions (VAS-Mood). No significant differ- } \\
\text { ence in PANAS scores across conditions. }\end{array}$ & 3 & 2 & 1 & 6 \\
\hline $\begin{array}{l}\text { Jay et al } \\
2006,2008 \\
(29,37)\end{array}$ & $\begin{array}{l}9 \text { male train } \\
\text { drivers } \\
51.3(6.4) \\
\text { years; } 42-63 \\
\text { years }\end{array}$ & $\begin{array}{l}\text { 80h operation } \\
\text { 8h-on/8h-off }\end{array}$ & $\begin{array}{l}P S G \\
S S Q\end{array}$ & $\begin{array}{l}\text { Comparison of sleep } \\
\text { environments (home } \\
\text { vs. crew van vs. hotel } \\
\text { layover) } \\
\text { Data collapsed across } \\
\text { shift rotation }\end{array}$ & $\begin{array}{l}\text { TST per sleep period was higher at home } \\
\text { followed by hotel layover then relay van } \\
\text { sites } \\
\text { After controlling for TST, SE, SOL, REM } \\
\text { were similar across sleep sites } \\
\text { SWS and subjective sleep quality were } \\
\text { lower in relay van compared to layover } \\
\text { sites }\end{array}$ & 3 & 0 & 0 & 3 \\
\hline $\begin{array}{l}\text { Kosmadop- } \\
\text { oulos et al, } \\
2014 \text { (40) }\end{array}$ & $\begin{array}{l}29 \text { healthy } \\
\text { males } \\
22.5(2.6) \text {. }\end{array}$ & $\begin{array}{l}\text { Forced desyn- } \\
\text { chrony study.13 } \\
\text { participants ad- } \\
\text { hered to a stan- } \\
\text { dard sleep-wake } \\
\text { schedule }(9.33 \mathrm{~h} \\
\text { TIB per } 28 \mathrm{~h}) \text { while } \\
16 \text { adhered to a } \\
\text { split-sleep sched- } \\
\text { ule }(4.67 \mathrm{~h} \text { TIB per } \\
\text { 14h). }\end{array}$ & $\begin{array}{l}\text { PVT } \\
\text { PSG } \\
\text { Core Body } \\
\text { Temperature } \\
\text { Subjective } \\
\text { predicted } \\
\text { performance }\end{array}$ & $\begin{array}{l}\text { Comparison of stan- } \\
\text { dard sleep-wake } \\
\text { schedule to split-sleep } \\
\text { schedule }\end{array}$ & $\begin{array}{l}\text { TST did not significantly differ across } \\
\text { conditions (regular sleep schedule = } \\
456.6 \pm 45.4 \text {, split-sleep schedule = } \\
475.4 \pm 28.0 \text { ). } \\
\text { No significant difference on any of the } \\
\text { PVT metrics across conditions (lapses, } \\
\text { slowest } 10 \% \text {, fastest } 10 \% \text {, reciprocal } \\
\text { reaction time). } \\
\text { No significant difference in subjec- } \\
\text { tive predicted performance between } \\
\text { conditions. }\end{array}$ & 3 & 2 & 0 & 5 \\
\hline $\begin{array}{l}\text { Lamond et } \\
\text { al 2005a, } \\
2005 b(25, \\
36)\end{array}$ & $\begin{array}{l}15 \text { male train } \\
\text { drivers } \\
46.6(4.9) y \text {; } \\
38-56 y\end{array}$ & $\begin{array}{l}\text { Two short (each } \\
<48 \mathrm{~h}) \text { operations } \\
8 \mathrm{~h}-\text { on/8h-off }\end{array}$ & $\begin{array}{l}\text { Actigraphy } \\
\text { PVT } \\
\text { SSS }\end{array}$ & $\begin{array}{l}\text { Comparison of shift } \\
\text { rotation (team one } \\
\text { commenced duty } \\
\text { at } 1700-2000 \mathrm{~h} \text { and } \\
\text { then recommenced at } \\
0400-1200 \mathrm{~h} \text { [early] } \\
\text { and team two at 2000- } \\
\text { 0400h [late]) }\end{array}$ & $\begin{array}{l}\text { TST was lower on the early rotation } \\
\text { Subjective alertness prior to a sleep } \\
\text { period, TIB and TST declined across } \\
\text { subsequent trip days on the early shift } \\
\text { rotation, but not late shift rotation } \\
\text { PVT performance was constant across } \\
\text { subsequent trip days on the early shift } \\
\text { rotation, but worse on the final on-duty } \\
\text { shift on the late shift rotation }\end{array}$ & 2 & 2 & 1 & 5 \\
\hline $\begin{array}{l}\text { Lützhöft et al } \\
2010(30)\end{array}$ & $\begin{array}{l}30 \text { watch } \\
\text { keepers } \\
29 \text { male, } 1 \\
\text { female } \\
45.8(11.7) \\
\text { years; } 27-60 \\
\text { years }\end{array}$ & $\begin{array}{l}\text { 6h-on/6h-off } \\
(\mathrm{n}=15) \text { and } 4 \mathrm{~h}- \\
\text { on/8h-off }(\mathrm{N}=15) \\
\text { Tours ranged } \\
\text { from } 4 \text { weeks to } 6 \\
\text { months }\end{array}$ & $\begin{array}{l}\text { KSS } \\
\text { Actigraphy } \\
\text { Blink duration } \\
\text { Reaction time }\end{array}$ & $\begin{array}{l}\text { Compare } 2 \text { watch (6h- } \\
\text { on/6-h off) system } \\
\text { with a } 3 \text { watch ( } 4 \mathrm{~h}- \\
\text { on/8h-off) system }\end{array}$ & $\begin{array}{l}\text { Sleepiness on night shift was higher on } \\
6 \mathrm{~h}-0 \mathrm{n} / 6 \mathrm{~h} \text { off schedule } \\
\text { Sleepiness increased more across } 6 \mathrm{~h} \\
\text { watch than } 4 \mathrm{~h} \text { watch } \\
\text { No differences in overall sleepiness, TST, } \\
\text { SE, reaction time, or blink duration } \\
\text { Sleep efficiency was low in both sched- } \\
\text { ules }(<80 \%) \\
\text { Shift length was routinely longer than } \\
\text { scheduled, especially for the } 6 \mathrm{~h}-0 \mathrm{n} / 6 \mathrm{~h}- \\
\text { off schedule }(6 \mathrm{~h} 48 \mathrm{~m} \pm 1 \mathrm{~h} 24 \mathrm{~m} \text { vs } 4 \mathrm{~h} 12 \mathrm{~m} \\
\pm 34 \mathrm{~m})\end{array}$ & 2 & 2 & 1 & 5 \\
\hline $\begin{array}{l}\text { Rutenfranz et } \\
\text { al } 1988 \text { (44) }\end{array}$ & $\begin{array}{l}\text { k8 watch } \\
\text { keepers and } \\
29 \text { day } \\
\text { workers } \\
\text { Age NR }\end{array}$ & $\begin{array}{l}\text { 4h-on/8h-off } \\
\text { watch } \\
\text { 24h watch } \\
\text { schedule }\end{array}$ & Sleep diary & $\begin{array}{l}\text { Compare sleep length } \\
\text { across different shift } \\
\text { schedules (04-08/16- } \\
20,12-16 / 00-04, \\
08-12 / 20-24 \text { and day } \\
\text { workers) }\end{array}$ & $\begin{array}{l}\text { Sleep duration was } 7.90 \mathrm{~h} \text { per } 24 \mathrm{~h} \text { for } \\
\text { those working } 1200 \mathrm{~h}-1600 \mathrm{~h} / 0000 \mathrm{~h}- \\
0400 \mathrm{~h}, 7.56 \mathrm{~h} \text { per } 24 \mathrm{~h} \text { for those working } \\
0400 \mathrm{~h}-0800 \mathrm{~h} / 1600 \mathrm{~h}-2000 \mathrm{~h} \text {, and } 7.20 \mathrm{~h} \\
\text { per } 24 \mathrm{~h} \text { for those working } 0800 \mathrm{~h}- \\
1200 \mathrm{~h} / 2000 \mathrm{~h}-2400 \mathrm{~h} \text {. Day workers aver- } \\
\text { aged } 7.51 \mathrm{~h} \text {. }\end{array}$ & 1 & 0 & 0 & 1 \\
\hline
\end{tabular}


Table 1. continued

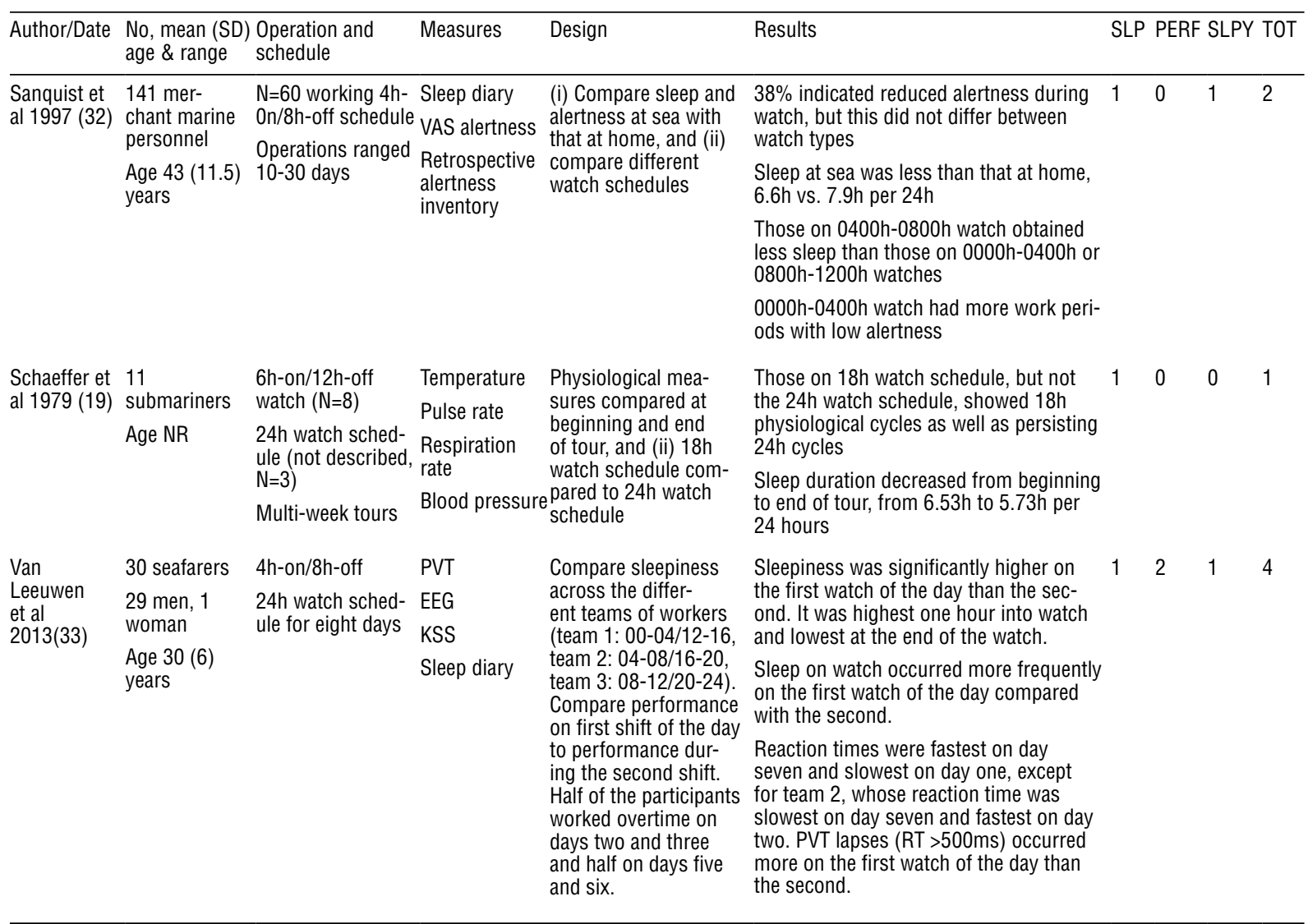

ules (17-19, 25-28, 30-32, 34, 35, 38-40); (ii) work/ rest schedules with daytime naps (24); and (iii) work/ rest schedules that incorporate a swing shift, "dog" shift, layover or disruption, typically in the middle of a roster schedule $(22,23,29,33,37)$. Five studies examined 8 hours-on/ 8 hours-off roster schedules, all of which were conducted with Australian train drivers (22, 25, 29, 36, 37 ), five examined 6 hours-on/6 hours-off shift schedules, predominantly in ship bridge officers and offshore fleet workers $(17,23,30,34,39)$, and nine examined 4 hours-on/8 hours-off shift schedules all among maritime watch keepers $(17,26-28,30-33,35)$. The remaining LWSW studies employed less common schedules. These included: 4 hours-on/4 hours-off and 4 hours-on/14 hours-off routines among laboratory volunteers simulating a shift work environment; 4 hours-on/12 hours-off, 2 hours-on/10 hours-off and 2 hours-on/12 hours-off routines among ship officers; 6 hours-on/12 hours-off routine among submariners; a split sleep schedule $(9.33$ hours wake/4.67 hours sleep opportunity) in a forced desynchrony study with volunteers; and an anchor night 4 hours or 8 hours sleep with distributed daytime naps among soldiers $(18,19,24,38,40)$.

\section{Design and research environment}

Eight of the studies identified in this review used a within-subject's design $(18,22,23,25,29,36-38)$, ten a between-subject's design $(17,24,26,27,30,31,34$, $35,39,40)$, and four employed a mixed within- and between-subjects design $(19,28,32,33)$. Seventeen studies were field-based (17-19, 22, 24-32, 34-37) and five used laboratory simulations $(23,33,38-40)$.

\section{Measures}

A notable feature of the studies identified in this review was the limited overlap of measures, which hindered comparisons. In order to summarize the methodological approaches and assess study quality, a simple scoring key was developed with higher scores indicating higher objectivity (scores are reported in the far right columns of table 1). Specifically, articles were assigned a score for sleep $(0=$ not measured; $1=$ diary; $2=$ actigraphy; $3=$ polysomnographic (PSG), performance $(0=$ not measured; $1=$ subjective measurements; $2=$ objective measurements) and sleepiness $(0=$ not measured; $1=$ subjective measurements; $2=$ objective measurements) 
measurement. The maximum possible score was seven points; however the maximum score achieved by any one study was six points while the modal score was two points. In general, studies focus on one or two domains with only a few more recent studies examining the gamut of sleep, sleepiness and performance, indicating a fertile ground for future research.

\section{Comparing LWSW schedules}

To help compare findings between LWSW studies, standardized scores for sleep, sleepiness, and performance were derived. Thirteen studies $(17,22,23,25,26,28-30$, $32,33,39,44)$ provided data on sleep time, along with sufficient schedule detail to calculate total sleep time per 24 hours, which included data for 25 different crews. In order to perform simple comparisons of average 24 hours total sleep time across roster types and crews, means and standard deviation (SD) from studies were pooled (weighted by sample size, such that each study mean and SD were multiplied by N) across crews to provide roster means and SD, and across studies for the 4 hours-on $/ 8$ hours-off rosters to provide crew means and SD within this roster. Analysis of variance (ANOVA) with simple posthoc comparisons was then conducted to assess differences between rosters and between crews within rosters. In this analysis, it is acknowledged that sleep measures differed between studies, with two studies using PSG $(29,39)$, four studies using actigraphy $(22,26,30,36)$ and the remaining studies using sleep diaries $(17,28,32,33,44)$.

Only 12 studies reported performance measures, and of these, 5 reported the psychomotor vigilance test (PVT) $(22,25,33,39,40)$, and the rest involved other measures of reaction time or vigilance $(18,24,30$, 38 ), processing speed and memory (27) and subjective performance reports $(34,40)$. Nine studies reported sleepiness measures, and of these, two reported visual analog scale (VAS) alertness $(27,32)$, five reported Karolinksa Sleepiness Scale (KSS) (17, 23, 30, 33, 39) and the others employed author-generated measures of sleepiness/alertness. Therefore, harvesting of performance or sleepiness/alertness measures from studies to compile for meta-analysis was deemed unfeasible and not pursued. Rather, patterns in performance and sleepiness are summarized in a narrative fashion following meta-analyses of the sleep data.

In order to facilitate testing of our specific hypotheses for the sleep data, a scoring system was constructed. Each crew within each schedule was initially scored using the following equation:

LWSW score = proportion of nadir on shift (scored 0-1) + proportion of sleep opportunity in WMZ (scored $0-1)+$ inconsistent timing of start and finish times from on 24 hours cycle to the next (scored yes $=1, n o=0)+$ work-to-rest ratio
Schedules involving (i) more work time during the circadian nadir, (ii) where more of the sleep opportunity falls during the WMZ, (iii) where the timing is inconsistent from one 24-hour cycle to the next, and (iv) the work-to-rest ratio is high will result in higher scores indicating greater likelihood of poor outcomes. For example, for a 00:00 hour crew on a 4 hours-on $/ 8$ hours-off schedule, LWSW score $=0+0.5+0+0.5$ with a total of 1, whereas for a 04:00 hour crew, LWSW score $=1+0.25+0+0.5$ with a total of 1.75 . We consequently used regression models to test the strength of each predictor, and then the overall score, in predicting the sleep time data. First, work time during the nadir, sleep during the WMZ, changing work/rest timing every 24 hours (yes/no) and work-to-rest schedule were regressed separately against total sleep time per 24 hours for each of the crews, and for each of the schedules (weighted by sample size). Next, a multiple regression was conducted containing all four predictors. Only significant predictors were included in the final model. Results of the multiple regression model were then used to identify significant predictors of total sleep time across studies.

\section{Results}

\section{Subgroup meta-analyses}

Differences in LWSW schedules and sleep. The shift schedules examined in this review (where schedule timing was provided) are illustrated in the top panel of figure 1. Within each schedule, each line represents a 24-hour period and each crew is represented on a different line, labelled by the start time of the first shift in that 24-hour period. The lower panel displays circadian fluctuations in subjective and objective sleepiness, respectively, and core body temperature, with the circadian nadir and WMZ overlaid across the two panels. Inspection of figure 1 shows a wide variation among shift systems in terms of the time from the beginning of one shift to the beginning of the next, length of shift, and the proportion of time spent on and off shift.

Figure 2 illustrates the mean daily sleep duration for 4 hours-on/8 hours-off, 8 hours-on/ 8 hours-off and 6 hours-on/ 6 hours-off studies, labelled according to the start time of the first shift for each crew.

Figure 2 illustrates the impact of the differing LWSW schedules on time spent asleep per 24 hours period. Pooled means across studies yielded an average of 6.9 (SD 1.2) hours per 24 hour period for the 4 hours-on/ 8 hours-off roster, compared to 5.6 (SD 1.0) hours for the 8 hourson/8 hours-off and 5.9 (SD 1.5) hours for the 6 hours-on/ 6 hours-off rosters. ANOVA indicated there were significant 
differences between rosters $\left(\mathrm{F}_{2,204}=27.453, \mathrm{P}<0.001\right)$, with post-hoc comparisons (LSD) revealing that those on 4 hours-on/ 8 hours-off rosters obtained significantly more sleep than those on 8 hours-on/ 8 hours-off or 6 hours-on $/ 6$ hours-off rosters $(\mathrm{P}<0.01$, with a mean difference of 1.3 hours and 1 hour respectively). Within the 4 hours-on/ 8 hours-off roster, the difference in sleep duration between crews approached significance $\left(\mathrm{F}_{2,142}=2.460, \mathrm{P}=0.089\right)$. Despite this, given the exploratory nature of this analysis, post-hoc comparisons were conducted, which revealed that crews starting at 04:00 hours (who were off-duty between 08:00-16:00 hours and between 20:00-04:00 hours each 24-hour period) obtained significantly less sleep than crews starting at 08:00 hours (and who were off-duty between 12:00-20:00 hours and between 00:00 08:00 hours each 24-hour period). Within the 8 hours- on $/ 8$ hours-off roster, there was a significant difference between studies $\left(\mathrm{F}_{3,49}=5.123, \mathrm{P}=0.004\right)$, with train drivers obtaining more sleep on the shorter (48 hours) versus longer (106 hours) return trip, but this may explained by the 8 -hour layover and 3-hour advance in the 8 hours-on $/ 8$ hours-off schedule obtained by train drivers on the longer return trip. Although limited to findings from one study by Eriksen et al (23), examination of the 6 hours-on/ 6 hours-off roster data revealed no significant differences between crews.

For each crew and roster schedule, figure 1 provides an illustration of the proportion of work time coincident with the circadian nadir and rest time with the WMZ. Figure 3 displays the LWSW scores for each crew and roster schedule, represented as a stacked proportion bar graph. The value for each LWSW component is
Figure 1. Upper panel: Sleep/rest (black) and work (white) schedules for the studies identified in this review where shift length and timing were provided, classified as $4 \mathrm{~h}-\mathrm{on} / 4 \mathrm{~h}-\mathrm{off}$, 8h-on/8h-off, 6h-on/6h-off or Irregular. For the regular roster types, each line represents a separate crew member, with time of first shift start indicated. ${ }^{\wedge}$ This shift crosses midnight, start time given as midnight for this $24 \mathrm{~h}$ period. For the irregular rosters $(\mathrm{M}, \mathrm{N})$, crew work a staggered start. Lower panel: Circadian fluctuation in subjective sleepiness ratings (Ratings), Multiple Sleep Latency Test time taken to fall asleep (MSLT) and core body temperature (data from Gradisar \& Lack, 2004). The circadian low point (nadir) and Wake Maintenance Zone (WMZ) are overlayed across the two panels. References - $A$ : Arendt et al 2006; B: Condon et al 1988; C: Lützhöft et al 2010; D: Rutenfranz et al 1998; E: Sanquist et al 1997; F: van Leeuwen et al 2013; G: Darwent et al 2008; H: Lamond et al 2005; I: Eriksen et al 2006; J: Härmä et al 2008; K: Lützhöft et al 2010; L: Jackson et al 2014; M: Hartley et al 1974; N: Colquhoun et al 1987; 0: Colquhoun et al 1968.

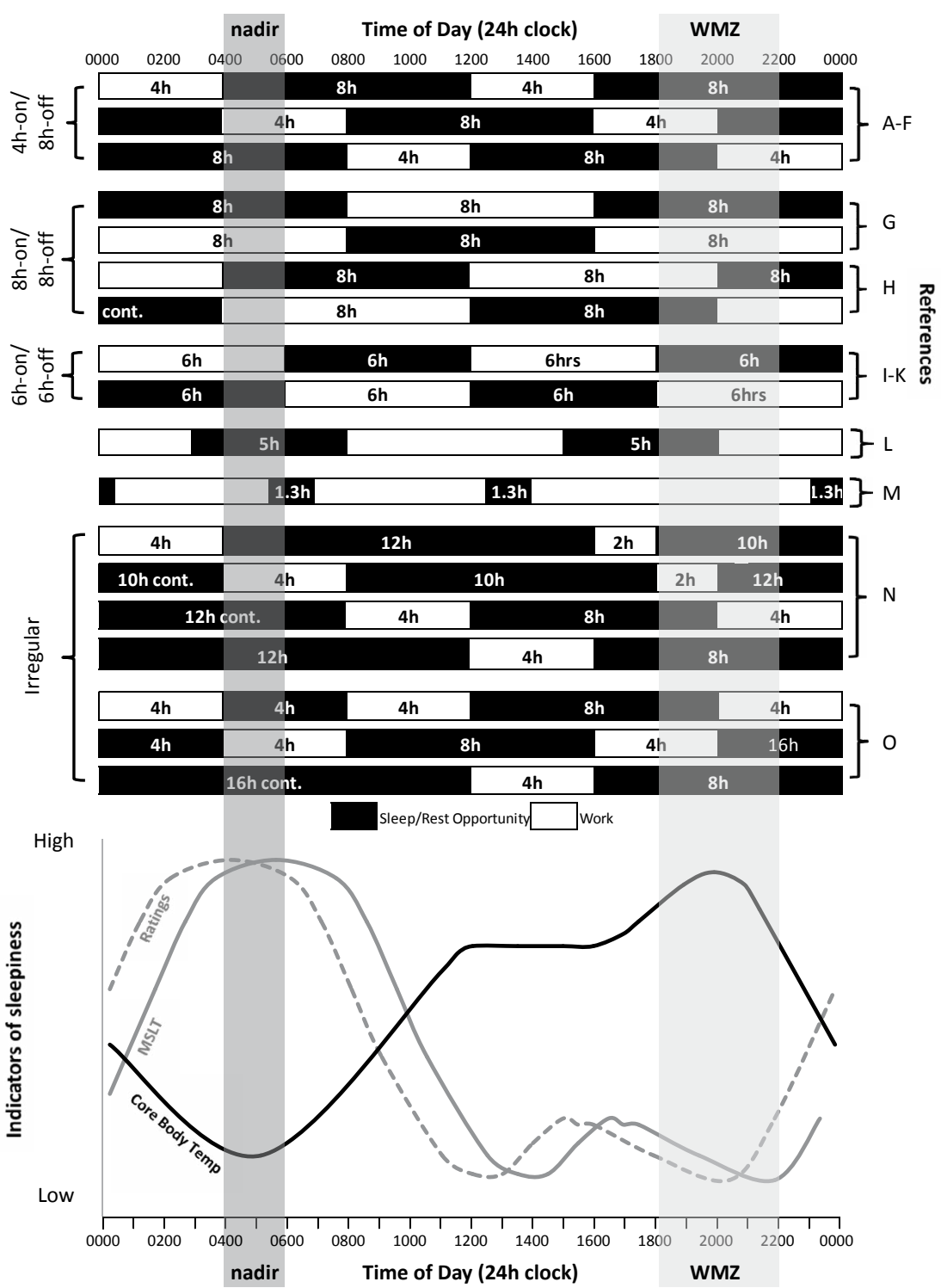


presented in different shading, and these are stacked such that the height of the bar represents the total score. Inspection of figure 3 indicates that sleep and performance deficits are greatest for the 8 hours-on $/ 8$ hours-off, followed by the 6 hours-on/ 6 hours-off then the 4 hours-on/ 8 hours-off schedules. It also reveals that crew start time has a strong impact (eg, scores are higher for 4 hours-on/8 hours-off crews starting at 04:00 hours compared to 00:00 hours and 08:00 hours).

Figure 4 presents results of the regression models of the relationship between the proportion of the nadir at work $\left(\mathrm{B}=-0.66\right.$, sterr $\left.=0.12, \mathrm{t}=5.6, \mathrm{P}<0.001, \mathrm{R}^{2}=0.08\right)$, the proportion of sleep in the $\mathrm{WMZ}(\mathrm{B}=0.39$, sterr= $0.30, \mathrm{t}=1.3, \mathrm{P}=0.19, \mathrm{R}^{2}=0.005$ ), whether the shift start and end times were consistent every 24 hours $(B=-2.00$, sterr $\left.=0.14, \mathrm{t}=14.1, \mathrm{P}<0.001, \mathrm{R}^{2}=0.35\right)$ and work-to-rest ratio $\left(\mathrm{B}=-1.82\right.$, sterr $\left.=0.23, \mathrm{t}=8.0, \mathrm{P}<0.001, \mathrm{R}^{2}=0.14\right)$ and the 24 hours total sleep time estimates shown in figure 2. All factors except proportion of sleep period in the WMZ were significant predictors of total sleep time in these univariate models, in the hypothesized direction.

The lower panel of figure 4 presents results of the multiple regression model. In the final model, only pro- portion of the circadian nadir spent at work $(\mathrm{B}=-0.51$, sterr $=0.10, \beta=-215, \mathrm{t}=-5.3, \mathrm{P}<0.001)$ and whether shift start and end times changed every 24 hours $(B=-1.92$, sterr $=0.14, \beta=-0.56, t=-13.9, \mathrm{P}<0.001)$ were significant in the final model, such that as these factors increased, sleep duration decreased. The scatterplot of the predicted values from the model (x-axis) against the 24 hours total sleep time values (y-axis) displays the model $\mathrm{R}^{2}$ of 0.39 (however, the skew in distribution of scores should be noted). This suggests that the crews that experience changing start times (ie, those on the 8 hours-on/ 8 hours-off roster) appear to be relatively disadvantaged in terms of sleep time, as are those who have a proportion of their nadir on shift (4 hours-on/8 hours-off roster, 04:00 hours crew; and 6 hours-on/6 hours-off roster, 00:00 hours crew).

\section{Narrative review}

Exploring variability in sleep duration. While shifts with greater sleep opportunities were associated with more sleep, sleep duration nevertheless varied substantially between studies - even among those with identical shift timing and duration $(17,23,26,30,33,34)$. Three

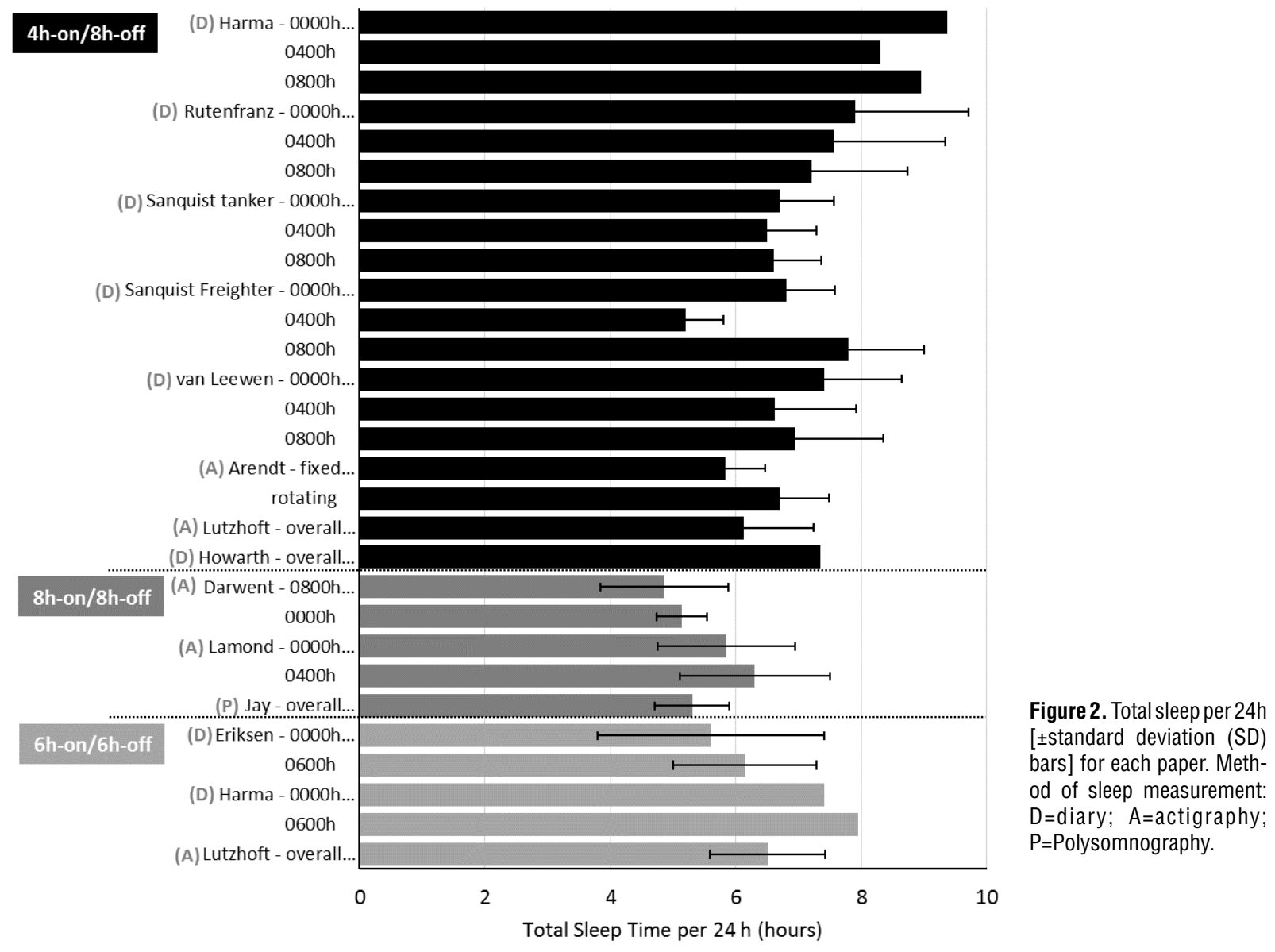




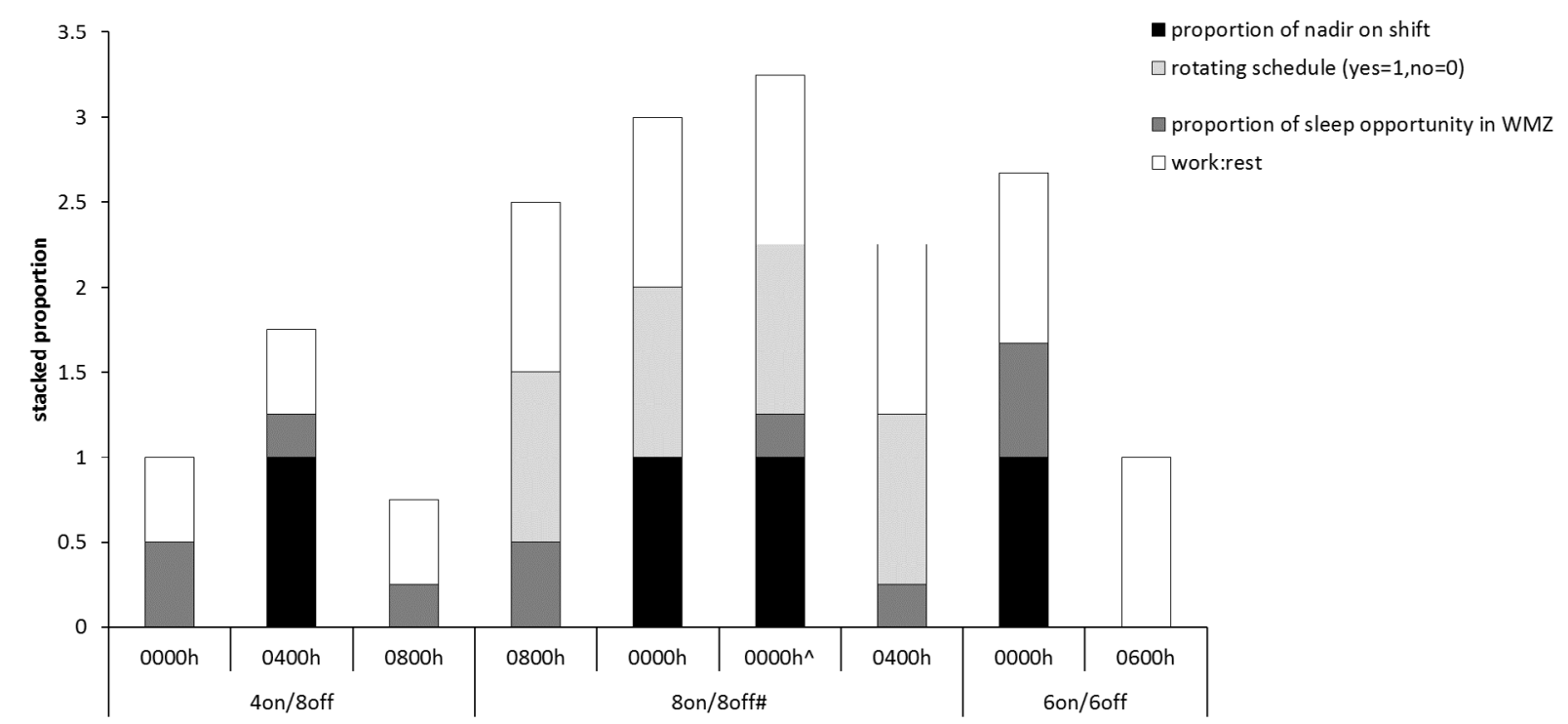

Figure 3. Hypothesized schedule-based factors contributing to sleep loss, sleepiness and performance impairment. Stacked bar graph showing proportion of nadir on shift (black), proportion of sleep period in Wake Maintenance Zone (WMZ) (grey), whether there are changes to the timing of work and rest periods every $24 \mathrm{~h}$, (yes/no), and work:rest ratio (white) [stacked proportion=proportion of nadir on shift $(0-1)+$ proportion of sleep opportunity in WMZ $(0-1)+24 \mathrm{~h} \mathrm{timing} \mathrm{changes} \mathrm{(yes=1,} \mathrm{no=0)} \mathrm{+} \mathrm{work:rest} \mathrm{ratio].} \mathrm{Notes:} \mathrm{\# scores} \mathrm{given} \mathrm{for} \mathrm{first} 24 \mathrm{~h}$ only for the $8 \mathrm{~h}-0 \mathrm{n} / 8 \mathrm{~h}-\mathrm{off}$. Due to the timing changes every $24 \mathrm{~h}$ for these rosters, in the second $24 \mathrm{~h}$, the scores for the 08:00h swap with the 00:00h, and the scores for the $\wedge$ 00:00h swap with the 04:00h. The scores for each crew for the $4 \mathrm{~h}-0 \mathrm{n} / 8 \mathrm{~h}-\mathrm{off}$ and $6 \mathrm{~h}-\mathrm{on} / 6 \mathrm{~h}-\mathrm{off}$ remain the same each $24 \mathrm{~h}$ period.

factors might account for this variability. First, sleep duration was related to how closely crews adhered to shift start and finish times $(30,34)$. Lutzhoft (30) found that watch keepers on a 6 hours-on/ 6 hours-off schedule worked an extra 48 minutes per shift, on average, which significantly eroded their daily opportunity for sleep. Second, sleep duration was related to the quality of LWSW sleep environment (eg, ship cabins, submarine bunks, mining "dongas" and train sleeping wagons). Underlying the importance of environmental factors for optimizing sleep, most studies reported that sleep length was shorter on operations compared to either home or layover $(22,25,29,32,36,45)$. In their large $(\mathrm{N}=362)$ survey of offshore fleet workers, Hansen et al (34) found the most frequently endorsed problems when sleeping at sea were noise $(55 \%)$, cabin vibration $(34 \%)$, cabin temperature/humidity $(30 \%)$, alarms $(29 \%)$, and poor bedding $(21 \%)$, all of which were more highly ranked than "bothered by shift work" (18\%). Most (81\%) did not report similar problems at home. The third factor affecting the reliability of sleep duration estimates and hence making it difficult to compare between studies is the lack of consistency in measures. Since diary measures tend to overestimate sleep duration compared to actigraphy and PSG (46), the lower sleep durations in the 8 hours-on/ 8 hours-off studies may be at least partially explained by the fact that they relied on objective measures of sleep, while studies examining other rosters commonly used diaries. Only three studies examined the impact of LWSW schedules on PSG sleep parameters $(29,39,40)$.

\section{LWSW and sleepiness}

Sleepiness was assessed in eight studies, and two trends were evident. First, higher levels of sleepiness tended to be reported in schedules with a longer time-at-work and shorter sleep opportunities. For example, both Härmä et al (17) and Lutzhoft et al (30) reported that sleepiness increased more rapidly and was higher among watch keepers working a 6 hours-on/6 hours-off (ie, 12-hour rest per 24 hours) compared to 4 hours-on/ 8 hours-off (ie, 16-hour rest per 24 hours) routine. This suggests that, on average, the 4 hours-on/ 8 hours-off rosters schedule is likely to be better for sleepiness than either the 6 hours-on/ 6 hours-off or 8 hours-on/ 8 hours-off rosters. The second trend was the strong circadian variation in sleepiness, with sleepiness peaking at night $(17,23$, $25,30,32,33,36)$, a finding that is well documented in the circadian literature (47). Taken together, these findings confirm those found in other studies of shift work and/or sleep restriction, namely, that greater sleepiness is experienced during the biological night $(47,48)$ with increased wakefulness $(9,49)$ and diminished sleep $(50)$.

\section{LWSW and performance}

Half of the studies identified included performance 

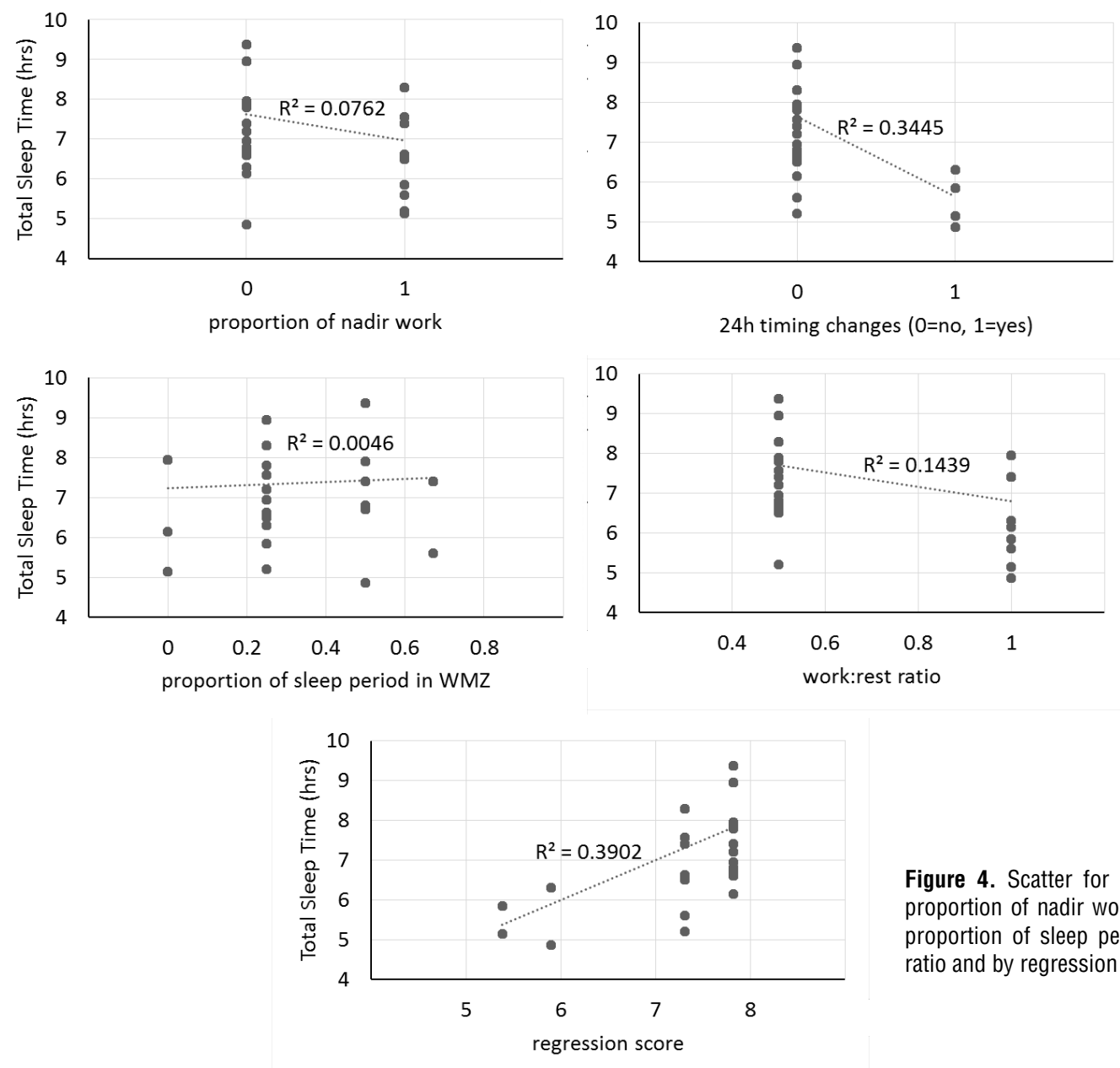

Figure 4. Scatter for sleep times (y-axis) by proportion of nadir work, $24 \mathrm{~h}$ timing changes, proportion of sleep period in WMZ, work:rest ratio and by regression score.

metrics, with most using the PVT. The PVT is well validated and generates measures of response time (how quickly an individual responds to a stimulus) and lapses of attention (the number of times an individual fails to respond to a stimulus for $>500 \mathrm{msec}$ ) (51). Two studies of Australian train drivers measured PVT lapses shortly before and after shifts $(22,25)$. Darwent and colleagues (22) report that both PVT response time and lapses were worse among drivers working the late (rested 08:00-16:00 hours, worked 16:00-24:00 hour) compared to early shift (worked 08:00-16:00 hours and rested 16:00-24:00 hours) roster. However, in both roster schedules, performance did not decline from the start to the end of the work cycle. In contrast, Lamond and colleagues (25) report that PVT performance was worse at the end of 8 hours-on/ 8 hours-off rosters, especially among train drivers working the late roster. Other performance measures, including measures of processing speed and memory (ie, the digit symbol substitution task) and visual vigilance, have also been utilized. The findings suggest that performance on these measures is worse on night shifts and when day sleep is restricted
$(24,52)$. Hartley demonstrated the potential benefit of splitting one nocturnal period of reduced sleep into three shorter periods spread across 24 hours, with improved performance on visual vigilance (24).

The influence of the circadian system on performance has also been examined by several LWSW groups. Colquhoun et al (53) examined the impact of working either a rotating 4 hours-on/4 hours-off or a fixed 4 hours-on/8 hours-off routine on vigilance and processing speed in a simulation exercise. Performance closely tracked the core body temperature rhythm and, moreover, a shift in the phase of the temperature rhythm was accompanied by a corresponding change in performance. Of interest, however, Kosmadopoulos et al (40) found that split sleep schedules (that roughly approximated a 6 hours-on/ 6 hours-off shift in a forced desynchrony protocol) resulted in a lessening of the typical performance deficits witnessed around the circadian nadir when compared to a schedule with one consolidated sleep opportunity. They conclude that splitting sleep may blunt both the circadian and sleep homeostatic contributions to performance deficits. 


\section{Discussion}

The present review has investigated the sleep, sleepiness, and performance implications of LWSW routines. Quantitative and narrative analyses of the literature suggest that sleep quality, sleepiness, and performance differ between schedules as well as crews working the same schedule but starting and finishing at different times in the 24-hour cycle. Taken together, the present results align with what we may hypothesize from previous literature, suggesting that schedules with day-to-day timing changes and with a high proportion of the work period during the circadian low, are likely to impact sleep negatively. In addition to these factors, the workto-rest ratio is likely to influence sleepiness, fatigue and performance. Consistent with this, findings suggest that 4 hours-on/ 8 hours-off may be superior to both 6 hourson/6 hours-off and 8 hours-on/ 8 hours-off shift schedules based on the outcomes considered in this review.

It should be noted that this does not take into account some of the unique considerations of the 4 hours-on $/ 8$ hours-off schedules, such as increased staff numbers and frequency of handover. Furthermore, all of the 8 hourson/8 hours-off rosters involved Australian relay train drivers, while all 6 hours-on/ 6 hours-off field studies involved the maritime sector. As such, systematic differences among individuals and working environments may account for some of the differences in risk indicators. The applied nature of the research question means that relevant research is always balancing ecological validity and experimental control. Convincing conclusions will ultimately be based on converging evidence from both field studies [eg, $(22,28,34)]$ and controlled simulations $[\mathrm{eg},(23,39,40)]$. Therefore, given the inherent differences in research environments, studies would benefit from improved consistency and quality in measures.

\section{LWSW and summary of operational considerations}

The operational considerations of the main LWSW schedules are summarized in table 2. Taken together, the literature support that LWSW shift schedules with a lower proportion of the nadir on shift, lower workto-rest ratio, and shift schedules that start and end at the same time every 24 hours may be preferable from a sleep, sleepiness, and performance perspective. Interestingly, the degree to which sleep periods occurred during the WMZ was not a significant predictor of sleep obtained per 24 hours. It may be that for the shift schedules reviewed, when sleep opportunities are split in this way, there is sufficient sleep pressure to override the circadian drive for wake during the WMZ. Indeed, in a recent forced desynchrony study involving restricted sleep opportunities, the expected lengthening in sleep onset latency near the circadian acrophase was extinguished, masking the "forbidden zone" for sleep (54).

Research into traditional shift work shows that, in addition to the length of shifts, the timing of shifts is important in determining outcomes associated with shift work. Even small changes to shift timing can result in improvements or decrements to performance and functioning. Tucker and colleagues found that a onehour difference in shift start time (07:00-06:00 hours) negatively impacted upon sleep (41). This is yet to be investigated in LWSW schedules. A notable feature of the start and end times of the various LWSW schedules examined in this review is their relative consistency with start and end times typically falling on even hours and rotations typically anchored at midnight or midday. This is especially notable in studies which have examined 4 hours-on/ 8 hours-off schedules, which all scheduled crews to start shifts at 00:00, 04:00, and 08:00 hours. Since risk factors can be apportioned differently between crews depending on the timing of shifts, there is a need to consider the possible impairments separately for each crew roster.

The considerations outlined in this review necessarily must be balanced by logistic and economic imperatives such as available workforce, salary costs, and the potential implications of increasing the number of staff handovers, which may in turn influence efficiency and safety. Some of these issues are summarized for each schedule in table 2. There are considerations particular to LWSW that arise from having more than one sleep period across 24 hours. For example, sufficient time must be given to individuals to prepare for sleep more than once per 24 hours. As such, two break periods incorporating the same time spent off-duty as one longer break period will not afford equal sleep opportunities. Multiple rest periods may also limit the contexts in which this type of shift work system can be implemented, more often being suitable in environments where the sleep quarters are in close proximity to the workplace in order to limit time spent travelling and maximize the sleep opportunity. Of the field studies examined, all encompassed situations where workers slept at the workplace, such as on trains, ships, and submarines. Further, while LWSW may offer promise, one limiting factor is the acceptability of LWSW to people working them. For example, Sanquist et al found virtually unanimous support among mariners for longer sleep and work periods, as opposed to having sleep split into separate episodes (32). This is likely to be another important reason that this type of shift work is only seen in limited contexts and where individuals are performing safety-critical tasks. To date, Härmä's group are the only LWSW researchers to have examined how individual differences affect sleep during LWSW. Other factors known to be predictive of 
Table 2. Summary of operational considerations for balancing limited wake shift work (LWSW) schedules based on predicted time of day considerations from the literature. These predictions are yet to be directly tested with these schedules.

\begin{tabular}{|c|c|c|}
\hline Roster & Advantages & Considerations \\
\hline 4h-on/8h-off & $\begin{array}{l}\text { 16h sleep opportunity every } 24 \mathrm{~h} \\
\text { Consistent times every } 24 \mathrm{~h}\end{array}$ & $\begin{array}{l}\text { Larger number of staff required } \\
\text { Increased number of crew handovers } \\
\text { While on average, circadian issues will be lower than the other two rosters, de- } \\
\text { pending on start times, certain crews may be relatively disadvantaged }\end{array}$ \\
\hline 8h-on/8h-off & $\begin{array}{l}\text { Fewer staff required than } 4-\text { on/8-off } \\
\text { Reduced number of crew handovers }\end{array}$ & $\begin{array}{l}\text { Changing timing every } 24 \mathrm{~h} \\
\text { The spread of circadian issues across crew is relatively even } \\
\text { Alternating } 16 \mathrm{~h} \text { and } 8 \mathrm{~h} \text { sleep opportunities every } 24 \mathrm{~h}\end{array}$ \\
\hline 6h-on/6h-off & $\begin{array}{l}\text { Fewer staff required than } 4 \mathrm{~h}-\mathrm{on} / 8 \mathrm{~h}-\mathrm{off} \\
\text { Reduced number of crew handovers compared to } \\
4 \mathrm{~h}-\mathrm{on} / 8 \mathrm{~h}-\mathrm{off} \\
12 \mathrm{~h} \text { sleep opportunity every } 24 \mathrm{~h} \\
\text { Consistent times every } 24 \mathrm{~h}\end{array}$ & $\begin{array}{l}\text { While on average, circadian issues will be lower than the } 8 \mathrm{~h}-\mathrm{on} / 8 \mathrm{~h}-\mathrm{off} \text { rosters, } \\
\text { depending on start times, certain crews may be relatively disadvantaged }\end{array}$ \\
\hline
\end{tabular}

general shift work tolerance have yet to be examined in LWSW (54-56).

\section{Review considerations}

There are a number of important limitations to this review that must be considered. For example, the review relied upon a relatively small number of studies that involved two main industries. As such, it is not possible to determine whether systematic differences in study population demographics, such as age, weight, health status, or smoking status, have confounded any differences between shift types. The majority of LWSW studies have been field-based with the advantages of ecological validity, but lacking the controls afforded by laboratory-based studies, including the impact of uncontrolled access to caffeine and other stimulants (53). As discussed previously, the effects of a particular schedule are likely to differ depending upon the specific situation and constraints of that environment. Operational environments are likely to differ according to the degree of proscription around sleep, use of time between shifts, characteristics of individuals working within the industry (eg, age, sex, and fitness), working conditions, sleeping conditions, light exposure, stress, and physical and cognitive workload $(55,56)$. Even within a workforce population there are likely to be differences between workers in terms of sleep, health, smoking status, medication and drug use, caffeine intake, and strategies employed to self-manage shift work (57). This is of particular concern when comparing 8 hours-on/ 8 hoursoff rosters, seen exclusively among long-haul train drivers, with those LWSW shifts, such as the 6 hours-on $/ 6$ hours-off and 4 hours-on/8 hours-off schedules worked by mariners. It is also likely that individuals who do not cope with the demands of LWSW schedules leave the industry. Thus, field studies may underestimate the negative effects of these schedules. The studies reviewed also frequently involve small samples and thus there is some concern regarding statistical power. The lack of uniform terminology to describe these shifts has also meant that identifying relevant literature is challenging and there is a risk that other, suitable studies have not been identified. Finally, it is possible that publication bias may mean that other studies showing non-significant results are not published.

The scoring system presented in this paper was not devised as a biomathematical model of sleep, sleepiness, or performance capacity. Indeed, there are a number of well-developed models that are designed for this purpose (eg, 58-61). Rather, this system was devised to provide a simple method allowing subgroup meta-analysis of the literature across studies that vary greatly in design (without the requirement for biomathematical modelling software). This suggested that the 8 hours-on/ 8 hours-off schedules for all crews may be more impairing from a sleep perspective than the 4 hours-on/ 8 hours-off or 6 hours-on/ 6 hours-off schedules. The regression model explained nearly $40 \%$ of the variance in 24 hours total sleep time values. It would have been ideal to validate the scoring system with a second dataset and/or regress it against other outcome values such as sleepiness or performance. Unfortunately, this was not possible with the available data. Further validation and refinement is unquestionably required, however, the current data provide proof of concept that this approach may be used successfully in future similar meta-analyses.

\section{Concluding remarks}

The present review identified 22 studies that examined a variety of LWSW roster schedules among $>1000$ participants. Results suggest that 4 hours-on/ 8 hours-off schedules offer more promise in terms of maximizing sleep, with likely benefits to sleepiness and performance. From 
the range of LWSW studies reviewed, several factors can be identified that are associated with improved sleep and performance. These include maximizing the proportion of rest time to work, strictly observing shift start and end times to avoid the erosion of rest breaks, and ensuring that rosters start and end at the same times each 24 hours. By contrast, other factors remain uncertain, in particular whether sleep opportunities spread across the day result in better performance than the same amount of sleep taken in one aliquot, and there are conflicting results from different studies [for example, Hartley (24) versus Mollicone (62)]. Furthermore, while LWSW rosters are an established work practice in a number of industries, further evidence is needed to conclude that these rosters lead to increased performance and/or sleep and less sleepiness due to diminished time-on-task and increased sleep opportunities when compared to mainstream rosters. This is a crucial area for future research.

\section{Funding}

The Bushfire Cooperative Research Centre funded this research.

\section{References}

1. Burgess PA. Optimal shift duration and sequence: recommended approach for short-term emergency response activations for public health and emergency management. Am J Public Health. 2007;97 Suppl 1:88-92. http://dx.doi. org/10.2105/AJPH.2005.078782.

2. Ferguson SA, Kennaway DJ, Baker A, Lamond N, Dawson D. Sleep and circadian rhythms in mining operators: limited evidence of adaptation to night shifts. Appl Ergon. 2012;43(4):695-701. http://dx.doi.org/10.1016/j. apergo.2011.11.003.

3. Hossain JL, Reinish LW, Heslegrave RJ, Hall GW, Kayumov L, Chung SA, et al. Subjective and objective evaluation of sleep and performance in daytime versus nighttime sleep in extended-hours shift-workers at an underground mine. J Occup Environ Med. 2004;46(3):212-26. http://dx.doi. org/10.1097/01.jom.0000117421.95392.31.

4. Parkes KR. Shift schedules on North Sea oil/gas installations: A systematic review of their impact on performance, safety and health. Safety Sci. 2012;50(7):1636-51. http://dx.doi. org/10.1016/j.ssci.2012.01.010.

5. Williamson AM, Feyer AM, Friswell R. The impact of work practices on fatigue in long distance truck drivers. Accid Anal Prev. 1996;28(6):709-19. http://dx.doi.org/10.1016/S00014575(96)00044-9.

6. Glazner LK. Factors related to injury of shiftworking fire fighters in the Northeastern United States. Safety Sci. 1996;21(3):255-63. http://dx.doi.org/10.1016/0925-
7535(95)00069-0.

7. Rosekind MR. Managing work schedules: an alertness and safety perspective. In: Kryger MH, Roth T, Dement WC, editors. Principles and Practice of Sleep Medicine. Philadelphia: Elsevier/Saunders; 2005. http://dx.doi. org/10.1016/B0-72-160797-7/50064-1.

8. Folkard S, Lombardi DA, Tucker PT. Shiftwork: safety, sleepiness and sleep. Ind Health. 2005;43(1):20-3. http:// dx.doi.org/10.2486/indhealth.43.20.

9. Van Dongen HP, Maislin G, Mullington JM, Dinges DF. The cumulative cost of additional wakefulness: dose-response effects on neurobehavioral functions and sleep physiology from chronic sleep restriction and total sleep deprivation. Sleep. 2003;26(2):117-26.

10. Knauth P, Landau K, Droge C, Schwitteck M, Widynski M, Rutenfranz J. Duration of sleep depending on the type of shift work. Int Arch Occup Environ Health. 1980;46(2):167-77. http://dx.doi.org/10.1007/BF00378195.

11. Dembe AE, Erickson JB, Delbos RG, Banks SM. Nonstandard shift schedules and the risk of job-related injuries. Scand $\mathrm{J}$ Work Environ Health. 2006;32(3):232-40. http://dx.doi. org/10.5271/sjweh.1004.

12. Åkerstedt T. Shift work and disturbed sleep/wakefulness. Occup Med (Lond). 2003;53(2):89-94. http://dx.doi. org/10.1093/occmed/kqg046.

13. Driscoll TR, Grunstein RR, Rogers NL. A systematic review of the neurobehavioural and physiological effects of shiftwork systems. Sleep Med Rev. 2007;11(3):179-94. http://dx.doi. org/10.1016/j.smrv.2006.11.001.

14. Borbely AA. Processes underlying sleep regulation. Horm Res. 1998;49(3-4):114-7. http://dx.doi.org/10.1159/000023156.

15. Lack LC, Lushington $K$. The rhythms of human sleep propensity and core body temperature. J Sleep Res. 1996;5(1):1-11. http://dx.doi.org/10.1046/j.1365-2869.1996.00005.x.

16. Strogatz SH, Kronauer RE, Czeisler CA. Circadian pacemaker interferes with sleep onset at specific times each day: role in insomnia. Am J Physiol. 1987;253(1 Pt 2):R172-8.

17. Härmä M, Partinen M, Repo R, Sorsa M, Siivonen P. Effects of $6 / 6$ and $4 / 8$ watch systems on sleepiness among bridge officers. Chronobiol Int. 2008;25(2):413-23. http://dx.doi. org/10.1080/07420520802106769.

18. Colquhoun WP, Watson KJ, Gordon DS. A shipboard study of a four-crew rotating watchkeeping system. Ergonomics. 1987;30(9):1341-52. http://dx.doi. org/10.1080/00140138708966028.

19. Schaefer KE, Kerr CM, Buss D, Haus E. Effect of 18-h watch schedules on circadian cycles of physiological functions during submarine patrols. Undersea Biomed Res. 1979;6 Suppl:S81-90.

20. Lavie P, Scherson A. Ultrashort sleep-walking schedule. I. Evidence of ultradian rhythmicity in "sleepability'. Electroencephalogr Clin Neurophysiol. 1981;52(2):163-74. http://dx.doi.org/10.1016/0013-4694(81)90164-4.

21. Shekleton JA, Rajaratnam SM, Gooley JJ, Van Reen E, Czeisler CA, Lockley SW. Improved neurobehavioral 
performance during the wake maintenance zone. J Clin Sleep Med. 2013;9(4):353-62. http://dx.doi.org/10.5664/ jesm.2588.

22. Darwent D, Lamond N, Dawson D. The sleep and performance of train drivers during an extended freight-haul operation. Appl Ergon. 2008;39(5):614-22. http://dx.doi.org/10.1016/j. apergo.2008.02.012.

23. Eriksen CA, Gillberg M, Vestergren P. Sleepiness and sleep in a simulated "six hours on/six hours off" sea watch system. Chronobiol Int. 2006;23(6):1193-202. http://dx.doi. org/10.1080/07420520601057981.

24. Hartley LR. A comparison of continuous and distributed reduced sleep schedules. Q J Exp Psychol. 1974;26(1):8-14. http://dx.doi.org/10.1080/14640747408400382.

25. Lamond N, Darwent D, Dawson D. Train drivers' sleep and alertness during short relay operations. Appl Ergon. 2005;36(3):313-8. http://dx.doi.org/10.1016/j. apergo.2005.01.011.

26. Arendt J, Middleton B, Williams P, Francis G, Luke C. Sleep and circadian phase in a ship's crew. J Biol Rhythms. 2006;21(3):214-21. http://dx.doi. org/10.1177/0748730405285278.

27. Condon R, Colquhoun P, Plett R, De Vol D, Fletcher N. Work at sea: a study of sleep, and of circadian rhythms in physiological and psychological functions, in watchkeepers on merchant vessels. IV. Rhythms in performance and alertness. Int Arch Occup Environ Health. 1988;60(6):405-11. http:// dx.doi.org/10.1007/BF00381387.

28. Howarth HD, Pratt JH, Tepas DI. Do maritime crew members have sleep disturbances? Int J Occup Environ Health. 1999;5(2):95-100. http://dx.doi.org/10.1179/ oeh.1999.5.2.95.

29. Jay SM, Dawson D, Lamond N. Train drivers'sleep quality and quantity during extended relay operations. Chronobiol Int. 2006;23(6):1241-52. http://dx.doi. org/10.1080/07420520601083409.

30. Lützhöft M, Dahlgren A, Kircher A, Thorslund B, Gillberg M. Fatigue at sea in Swedish shipping-a field study. Am J Ind Med. 2010;53(7):733-40. http://dx.doi.org/10.1002/ajim.20814.

31. Rutenfranz J, Plett R, Knauth P, Condon R, De Vol D, Fletcher $\mathrm{N}$, et al. Work at sea: a study of sleep, and of circadian rhythms in physiological and psychological functions, in watchkeepers on merchant vessels. II. Sleep duration, and subjective ratings of sleep quality. Int Arch Occup Environ Health. 1988;60(5):331-9. http://dx.doi.org/10.1007/BF00405666.

32. Sanquist TF, Raby M, Forsythe A, Carvalhais AB. Work hours, sleep patterns and fatigue among merchant marine personnel. J Sleep Res. 1997;6(4):245-51. http://dx.doi.org/10.1111/ j.1365-2869.1997.00245.x.

33. van Leeuwen WM, Kircher A, Dahlgren A, Lützhöft M, Barnett M, Kecklund G, et al. Sleep, Sleepiness, and Neurobehavioral Performance While on Watch in a Simulated 4 Hours on/8 Hours off Maritime Watch System. Chronobiol Int. 2013;13(9):1108-15. http://dx.doi.org/10.3109/0742052 8.2013.800874.
34. Hansen JH, Holmen IM. Sleep disturbances among offshore fleet workers: a questionnaire-based survey. Int Marit Health. 2011;62(2):123-30.

35. Condon R, Knauth P, Klimmer F, Colquhoun P, Herrmann H, Rutenfranz J. Adjustment of the oral temperature rhythm to a fixed watchkeeping system on board ship. Int Arch Occup Environ Health. 1984;54(2):173-80. http://dx.doi. org/10.1007/BF00378520.

36. Lamond N, Darwent D, Dawson D. How well do train driver's sleep in relay vans? Ind Health. 2005;43(1):98-104. http:// dx.doi.org/10.2486/indhealth.43.98.

37. Jay SM, Dawson D, Ferguson SA, Lamond N. Driver fatigue during extended rail operations. Appl Ergon. 2008;39(5):6239. http://dx.doi.org/10.1016/j.apergo.2008.01.011.

38. Colquhoun WP, Blake MJ, Edwards RS. Experimental studies of shift-work I: A comparison of 'rotating' and 'stabilized' 4-hour shift systems. Ergonomics. 1968;11(5):437-53. http:// dx.doi.org/10.1080/00140136808930993.

39. Jackson ML, Banks S, Belenky G. Investigation of the effectiveness of a split sleep schedule in sustaining sleep and maintaining performance. Chronobiol Int. 2014;31(10):1218 30. http://dx.doi.org/10.3109/07420528.2014.957305.

40. Kosmadopoulos A, Sargent C, Darwent D, Zhou X, Dawson D, Roach GD. The effects of a split sleep-wake schedule on neurobehavioural performance and predictions of performance under conditions of forced desynchrony. Chronobiol Int. 2014;31(10):1209-17. http://dx.doi.org/10.3109/07420528. 2014.957763 .

41. Tucker P, Smith L, Macdonald I, Folkard S. The impact of early and late shift changeovers on sleep, health, and well-being in 8- and 12-hour shift systems. J Occup Health Psychol. 1998;3(3):265-75. http://dx.doi.org/10.1037/10768998.3.3.265.

42. Smith L, Folkard S, Tucker P, Macdonald I. Work shift duration: a review comparing eight hour and 12 hour shift systems. Occup Environ Med. 1998;55(4):217-29. http:// dx.doi.org/10.1136/oem.55.4.217.

43. Pilcher JJ, Lambert BJ, Huffcutt AI. Differential effects of permanent and rotating shifts on self-report sleep length: a meta-analytic review. Sleep. 2000;23(2):155-63.

44. Rutenfranz J, Plett R, Knauth P, Condon R, De Vol D, Fletcher $\mathrm{N}$, et al. Work at sea: a study of sleep, and of circadian rhythms in physiological and psychological functions, in watchkeepers on merchant vessels. II. Sleep duration, and subjective ratings of sleep quality. Int Arch Occup Environ Health. 1988;60(5):331-9. http://dx.doi.org/10.1007/BF00405666.

45. Gander P, van den Berg M, Signal L. Sleep and sleepiness of fishermen on rotating schedules. Chronobiol Int. 2008;25(2):389-98. http://dx.doi. org/10.1080/07420520802106728.

46. Saksvik IB, Bjorvatn B, Harvey AG, Waage S, Harris A, Pallesen S. Adaptation and readaptation to different shift work schedules measured with sleep diary and actigraphy. J Occup Health Psychol. 2011;16(3):331-44. http://dx.doi. org/10.1037/a0022770. 
47. Monk TH. Subjective ratings of sleepiness--the underlying circadian mechanisms. Sleep. 1987;10(4):343-53.

48. Babkoff H, Caspy T, Mikulincer M. Subjective sleepiness ratings: the effects of sleep deprivation, circadian rhythmicity and cognitive performance. Sleep. 1991;14(6):534-9.

49. Belenky G, Wesensten NJ, Thorne DR, Thomas ML, Sing HC, Redmond DP, et al. Patterns of performance degradation and restoration during sleep restriction and subsequent recovery: a sleep dose-response study. J Sleep Res. 2003;12(1):1-12. http://dx.doi.org/10.1046/j.1365-2869.2003.00337.x.

50. Dinges DF, Pack F, Williams K, Gillen KA, Powell JW, Ott GE, et al. Cumulative sleepiness, mood disturbance, and psychomotor vigilance performance decrements during a week of sleep restricted to 4-5 hours per night. Sleep. 1997;20(4):267-77.

51. Dorrian J, Rogers NL, Dinges DF. Psychomotor vigilance performance: Neurocognitive assay sensitive to sleep loss: Marcel Dekker; 2005.

52. Lieberman HR, Niro P, Tharion WJ, Nindl BC, Castellani JW, Montain SJ. Cognition during sustained operations: comparison of a laboratory simulation to field studies. Aviat Space Environ Med. 2006;77(9):929-35.

53. Colquhoun WP, Blake MJF, Edwards BS. Experimental Studies of Shift-Work II: Stabilized 8-hour Shift Systems. Ergonomics. 1968;11(6):527-46. http://dx.doi. org/10.1080/00140136808931006.

54. Sargent C, Darwent D, Ferguson SA, Kennaway DJ, Roach GD. Sleep restriction masks the influence of the circadian process on sleep propensity. Chronobiol Int. 2012;29(5):56571. http://dx.doi.org/10.3109/07420528.2012.675256.

55. Kantermann T, Wehrens SM, Ulhoa MA, Moreno C, Skene DJ. Noisy and individual, but doable: shift-work research in humans. Prog Brain Res. 2012;199:399-411. http://dx.doi. org/10.1016/B978-0-444-59427-3.00022-8.

56. Rajaratnam SM, Arendt J. Health in a 24-h society. The Lancet. 2001;358(9286):999-1005. http://dx.doi.org/10.1016/S01406736(01)06108-6.

57. Meloni M, Setzu D, Del Rio A, Campagna M, Cocco P. QTc interval and electrocardiographic changes by type of shift work. Am J Ind Med. 2013;56(10):1174-9. http://dx.doi. org/10.1002/ajim.22207.

58. Fletcher A. Staying safe in the jungles of Borneo: five studies of fatigue and cultural issues in remote mining projects. Ind Health. 2010;48(4):406-15. http://dx.doi.org/10.2486/ indhealth.MSSW-04.

59. Dawson D, Ian Noy Y, Härmä M, Åkerstedt T, Belenky G. Modelling fatigue and the use of fatigue models in work settings. Accid Anal Prev. 2011;43(2):549-64. http://dx.doi. org/10.1016/j.aap.2009.12.030.

60. Folkard S, Åkerstedt T. Towards a model for the prediction of alertness and/or fatigue on different sleep/wake schedules. Contemporary advances in shiftwork research: Theoretical and practical aspects in the late eighties. 1987:231-40.

61. Hursh SR, Redmond DP, Johnson ML, Thorne DR, Belenky G, Balkin TJ, et al. Fatigue models for applied research in warfighting. Aviat Space Environ Med. 2004;75(3 Suppl):A44-53; discussion A4-60.

62. Mollicone DJ, Van Dongen HPA, Rogers NL, Dinges DF. Response Surface Mapping of Neurobehavioral Performance: Testing the Feasibility of Split Sleep Schedules for Space Operations. Acta Astronaut. 2008;63(7-10):833-40. http:// dx.doi.org/10.1016/j.actaastro.2007.12.005.

Received for publication: 19 December 2013 